\title{
Non-Uniformly Loaded Row of Moving, Antiplane Shear Cracks in One-Dimensional Piezoelectric Quasicrystals
}

\author{
G.E. Tupholme* \\ School of Engineering and Informatics, University of Bradford, Bradford BD7 1DP, UK \\ (Received March 28, 2018; in final form September 17, 2018)
}

\begin{abstract}
An antiplane shear deformation in one-dimensional piezoelectric quasicrystals created by a row of collinear cracks subject to non-uniform phonon, phason and electric displacement loads is analyzed using a generalized technique of dislocation layers. Closed-form expressions are derived for the components of the phonon and phason stress and electric displacement fields. Their variations with angle near a crack tip are demonstrated and the corresponding phonon and phason stress and electric displacement intensity factors are deduced. Previously constantly loaded rows of cracks in various media have been studied. But no explicit expressions for the field components at a general point nor their angular variations were presented for them; even in classical isotropic elastic solids. The material considered here is the currently most general that is amenable to such an investigation and some of the analogous results for non-quasicrystals or non-piezoelectric or purely elastic solids or stationary cracks that can be deduced as particular cases are outlined. Some illustrative numerical results are graphically presented.
\end{abstract}

DOI: 10.12693/APhysPolA.134.1144

PACS/topics: $61.44 . \mathrm{Br}, 62.20 . \mathrm{mt}, 61.72 . \mathrm{Lk}, 46.50 .+\mathrm{a}$

\section{Introduction}

There has been continuing interest during the last century in the fundamental analysis of cracks of various configurations within solid media since the pioneering work of Griffith [1]. Sneddon and Lowengrub [2] derived the stress-intensity factor near a crack tip in a row of stationary strip cracks subject to constant loads in a classical isotropic solid and corresponding analyses for more general materials have followed. However they do not present explicit closed-form expressions for the general components of the fields created; but only on the axis line of the constantly-loaded cracks. The components of the elastic, electric and magnetic fields and the corresponding intensity factors of a row of moving, constantlyloaded, collinear Griffith cracks in magnetoelectroelastic solids were investigated by Tupholme [3]. Analogously, Tupholme [4] derived closed-form expressions for the phonon and phason stress components of a similar row of cracks moving in one-dimensional hexagonal quasicrystals.

Here for a non-uniformly loaded row of moving cracks, an extended dislocation layer technique is used to deduce closed-form representations for the phonon, phason and electric displacement field components and intensity factors in one-dimensional piezoelectric quasicrystals. These appear to be the most modern advanced materials for which at present such comprehensive analyses can be derived. As particular cases, the previously-unavailable analogous results within some purely piezoelectric, or quasicrystalline or anisotropic or isotropic elastic media, and of course for stationary cracks, can be deduced.

\footnotetext{
*e-mail: g.e.tupholme@bradford.ac.uk
}

Much attention has been devoted to studying both theoretically and experimentally the behaviour of quasicrystals, since their discovery was initially reported by Shechtman et al. [5] in 1984. The technologies of their preparation and industrial usage and exploitation of the desirable, inherent effects of piezoelectric coupling are continually expanding.

It is crucially important that a portfolio of benchmark fundamental exact solutions is produced of a variety of boundary value problems and especially those related to defects in such materials, since it has been observed experimentally that quasicrystals are intrinsically brittle and thus prone to cracking. This greatly assists in providing the necessary checks upon numerical/experimental investigations of more complex practically-realistic situations that cannot be studied analytically.

Within a continuum mechanics framework, the equations governing the components of the phonon and phason fields in a linear theory of quasicrystals are now wellestablished and related research continues to attract considerable attention. Convenient accounts of this are provided by, for example, Fan et al. [6], Fan [7, 8] and Ding et al. [9] and their included references. The 56 presentations at the most recent 13th International Conference on Quasicrystals (ICQ13) in 2016 were interestingly summarized by Yadav [10].

The analogous three-dimensional equations governing the physical response of quasicrystals with piezoelectric effects were generated by Altay and Dökmeci [11] using a unified variational principle. Henceforth techniques have been developed for addressing boundary value problems in piezoelectric quasicrystals.

Wang and Pan [12] employed the underlying investigation of the physical property tensors of Li and Liu [13] to determine elementary representations for the electroelastic field components of a screw dislocation moving uni- 
formly in one-dimensional hexagonal piezoelectric quasicrystals. Stroh's generalized formalism was applied by Yang et al. [14] to obtain and numerically investigate the electric-elastic field of a stationary straight dislocation.

Three-dimensional general solutions to assist in static problems in hexagonal piezoelectric quasicrystals were derived by Li et al. [15] using operator theory. Further, Yu et al. [16] adopted operator and complex variable techniques to give general solutions which were applied using a semi-inverse method to consider a stationary constantly-loaded crack in a one-dimensional hexagonal piezoelectric quasicrystal. Solutions for an antiplane uniformly-loaded elliptical cavity were obtained by $\mathrm{Yu}$ et al. [17].

During 2016-18, the flourishing interest in piezoelectric effects of one-dimensional quasicrystals is demonstrated by ongoing publications. Zhang et al. [18] presented Green's functions for bi-materials of piezoelectric quasicrystals. A three-phase cylinder model was proposed by Guo and Pan [19] for quasicrystal composites of onedimensional hexagonal piezoelectric quasicrystals. Fan et al. [20] investigated fundamental solutions in terms of discontinuities in the phonon and phason displacements and electric potential for three-dimensional cracks. By methods of functions of complex variables and conformal mappings, Guo et al. [21] studied an elliptical inclusion in a composite matrix and Yang and $\mathrm{Li}$ [22] considered a circular hole with a straight crack. As limiting cases, their results yield those for a stationary Griffith crack subjected to uniform loads. Tupholme $[23,24]$ determined closed-form representations of the components of the fields around a moving non-uniformly loaded shear crack in an infinite extent and also in a half-space of onedimensional hexagonal piezoelectric quasicrystals. The antiplane problem of an elliptic hole with two asymmetric cracks was analyzed by Yang et al. [25] with the aid of conformal mappings and a Stroh-like formalism. Most recently, Zhou and Li [26] used Fourier transforms and triple and singular integral equations to consider a one-dimensional hexagonal piezoelectric quasicrystal strip with two collinear, stationary, constantly-loaded, antiplane cracks at its mid-plane. Expressions were given for the field components on the axis of the cracks.

In Sect. 2, the basic physical situation under consideration is outlined and the general three-dimensional governing equations of deformations of piezoelectric quasicrystals are presented. A summary is given of the appropriate constitutive equations for one-dimensional hexagonal piezoelectric quasicrystals with point group $6 \mathrm{~mm}$. The concept of a moving "piezoelectric quasicrystal screw dislocation" which inspired the current analysis is described in Sect. 3, alongside the phonon, phason and electric field components which it creates. The extension of the dislocation layer method is then used in Sect. 4 to determine the solution of the non-uniformly loaded, moving row of cracks problem in detail. In Sects. 5, 6, and 7 , respectively, the results for a non-uniformly loaded, stationary row of cracks in piezoelectric quasicrystals, a non-uniformly loaded, moving row of cracks in hexagonal piezoelectric crystals and a non-uniformly loaded, moving row of cracks in isotropic elastic solids are deduced as special cases. Finally, in Sect. 8, the main details of this study are summarized.

\section{Underlying equations governing piezoelectric quasicrystals and stipulation of the cracks' problem}

Altay and Dökmeci [11] summarized in variationally invariant and differential forms the fundamental threedimensional equations that govern the phonon, phason and quasi-static electric field components of a deformation in a linearized theory of piezoelectric quasicrystal continua. By exploiting the Einstein summation convention for repeated suffices $i, j, k, l=1,2,3$ relative to fixed rectangular Cartesian coordinates $\left(x_{1}, x_{2}, x_{3}\right)$ and partial differentiation with respect to $x_{p}$ being indicated by a $p$ following a comma for $p=i, j, k, l$, the overriding quasi-static equilibrium equations and constitutive equations of such materials when not subject to any body forces or electric charge densities can be expressed elegantly as

$$
\begin{aligned}
& \sigma_{i j, i}=0, \quad H_{i j, i}=0, \quad D_{i, i}=0, \\
& \sigma_{i j}=c_{i j k l}\left(u_{k, l}+u_{l, k}\right) / 2+R_{i j k l} w_{k, l}-e_{k i j} E_{k}, \\
& H_{i j}=R_{k l i j}\left(u_{k, l}+u_{l, k}\right) / 2+K_{i j k l} w_{k, l}-e_{k i j}^{\prime} E_{k}, \\
& D_{i}=e_{k i j}\left(u_{j, k}+u_{k, j}\right) / 2+e_{k i j}^{\prime} w_{j, k}-\varepsilon_{i j} E_{j} .
\end{aligned}
$$

$\sigma_{i j}, u_{i}, H_{i j}, w_{i}, D_{i}$ and $E_{i}$, respectively, represent the components of the phonon stress and displacement, the phason stress and displacement and the electric displacement and field, with $c_{i j k l}, R_{i j k l}, K_{i j k l}, e_{i j k}, e_{i j k}^{\prime}$ and $\varepsilon_{i j}$, respectively, being the phonon elastic, the phononphason coupling, the phason elastic, the phonon and phason piezoelectric and the dielectric constants.

Under consideration here is a one-dimensional hexagonal piezoelectric quasicrystal medium of point group $6 \mathrm{~mm}$ of uniform density, $\rho$, that is in a stress-free undisturbed state of reference initially. The $x-y$ plane is chosen to coincide with its periodic plane and the positive $z$-axis identifies its quasiperiodic poling direction, relative to a fixed Cartesian coordinate system, $(x, y, z)$.

The mode III fracture problem considered involves an embedded periodic, infinite, collinear sequence of moving, antiplane Griffith-strip shear cracks of constant equal width $2 a$ with non-uniform phonon, phason and electrical loads applied at infinity.

The constitutive equations relating the components $u_{X}, \sigma_{X Y}$ and $\varepsilon_{X Y}$ of the phonon displacement vector and stress and strain tensors, and $w_{X}, H_{z X}$ and $w_{z X}$ of the phason displacement vector and stress and strain tensors, and $D_{X}$ and $E_{X}$ of the electric displacement and field vectors, for $X$ and $Y=x, y$ or $z$, which are generated within the piezoelectric quasicrystal can be expressed in matrix notation as 


$$
\begin{aligned}
& {\left[\begin{array}{c}
\sigma_{x x} \\
\sigma_{y y} \\
\sigma_{z z} \\
\sigma_{y z} \\
\sigma_{x z} \\
\sigma_{x y} \\
H_{z z} \\
H_{z x} \\
H_{z y}
\end{array}\right]=\left[\begin{array}{ccccccccc}
c_{11} & c_{12} & c_{13} & 0 & 0 & 0 & R_{1} & 0 & 0 \\
c_{12} & c_{11} & c_{13} & 0 & 0 & 0 & R_{1} & 0 & 0 \\
c_{13} & c_{13} & c_{33} & 0 & 0 & 0 & R_{2} & 0 & 0 \\
0 & 0 & 0 & 2 c_{44} & 0 & 0 & 0 & 0 & R_{3} \\
0 & 0 & 0 & 0 & 2 c_{44} & 0 & 0 & R_{3} & 0 \\
0 & 0 & 0 & 0 & 0 & c_{11}-c_{12} & 0 & 0 & 0 \\
R_{1} & R_{1} & R_{2} & 0 & 0 & 0 & K_{1} & 0 & 0 \\
0 & 0 & 0 & 0 & 2 R_{3} & 0 & 0 & K_{2} & 0 \\
0 & 0 & 0 & 2 R_{3} & 0 & 0 & 0 & 0 & K_{2}
\end{array}\right]\left[\begin{array}{c}
\varepsilon_{x x} \\
\varepsilon_{y y} \\
\varepsilon_{z z} \\
\varepsilon_{y z} \\
\varepsilon_{x z} \\
\varepsilon_{x y} \\
w_{z z} \\
w_{z x} \\
w_{z y}
\end{array}\right]-\left[\begin{array}{ccc}
0 & 0 & e_{31} \\
0 & 0 & e_{31} \\
0 & 0 & e_{33} \\
0 & e_{15} & 0 \\
e_{15} & 0 & 0 \\
0 & 0 & 0 \\
0 & 0 & e_{33}^{\prime} \\
e_{15}^{\prime} & 0 & 0 \\
0 & e_{15}^{\prime} & 0
\end{array}\right]\left[\begin{array}{c}
E_{x} \\
E_{y} \\
E_{z}
\end{array}\right],} \\
& {\left[\begin{array}{c}
D_{x} \\
D_{y} \\
D_{z}
\end{array}\right]=\left[\begin{array}{ccccccccc}
0 & 0 & 0 & 0 & 2 e_{15} & 0 & 0 & e_{15}^{\prime} & 0 \\
0 & 0 & 0 & 2 e_{15} & 0 & 0 & 0 & 0 & e_{15}^{\prime} \\
e_{31} & e_{31} & e_{33} & 0 & 0 & 0 & e_{33}^{\prime} & 0 & 0
\end{array}\right]\left[\begin{array}{c}
\varepsilon_{x x} \\
\varepsilon_{y y} \\
\varepsilon_{z z} \\
\varepsilon_{y z} \\
\varepsilon_{x z} \\
\varepsilon_{x y} \\
w_{z z} \\
w_{z x} \\
w_{z y}
\end{array}\right]+\left[\begin{array}{ccc}
\varepsilon_{11} & 0 & 0 \\
0 & \varepsilon_{11} & 0 \\
0 & 0 & \varepsilon_{33}
\end{array}\right]\left[\begin{array}{c}
E_{x} \\
E_{y} \\
E_{z}
\end{array}\right]}
\end{aligned}
$$

where

$$
\varepsilon_{X Y}=\frac{1}{2}\left(\frac{\partial u_{X}}{\partial Y}+\frac{\partial u_{Y}}{\partial X}\right), \quad w_{z X}=\frac{\partial w_{z}}{\partial X} .
$$

With $i$ and $j$ taking integer values and utilizing the classical Voigt contracted notation, the constants involved are the phonon elastic moduli, $c_{i j}$, the phason elastic moduli, $K_{i}$, the phonon-phason coupling elastic moduli, $R_{i}$, the piezoelectric moduli, $e_{i j}$ and $e_{i j}^{\prime}$, and the dielectric moduli, $\varepsilon_{i j}$.

The cracks are assumed to be uniformly moving in their own planes parallel to their axes with a constant speed of propagation $v$, so that at time $t$ they are centred at $x=v t, v t \pm 2 h, v t \pm 4 h, \ldots$, on the $y=0$ plane. They thus occupy the regions

$$
\begin{aligned}
& R_{t}=\{(x, y, z): v t-a+2 n h<x<v t+a+2 n h, \\
& y=0, \quad-\infty<z<\infty\},
\end{aligned}
$$

within the intervals

$$
\begin{aligned}
& S_{t}=\{(x, y, z): v t+(2 n-1) h<x<v t+(2 n+1) h, \\
& -\infty<z<\infty\},
\end{aligned}
$$

for $n=0, \pm 1, \pm 2 \ldots$, as illustrated in Fig. 1 .

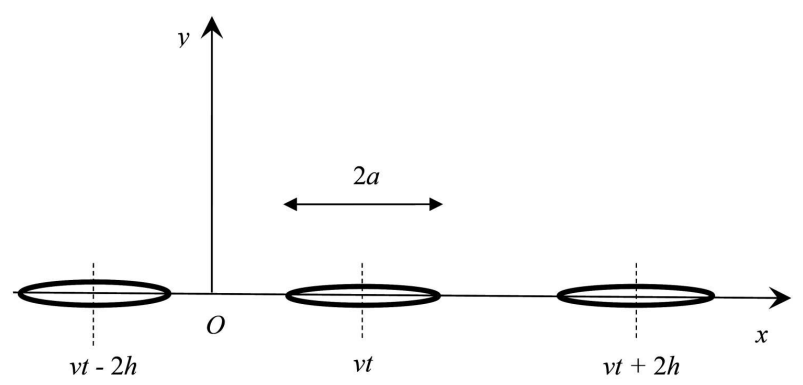

Fig. 1. Row of strip cracks moving in the $x$-direction with constant speed $v$.
It is expedient to introduce a moving coordinate, $\xi$, defined as:

$$
\xi=x-v t
$$

and express the electric field vector, $\boldsymbol{E}$, in terms of an electric potential, $\varphi$, which are related by

$$
\boldsymbol{E}=-\nabla \varphi=\left(\frac{\partial \varphi}{\partial x}, \frac{\partial \varphi}{\partial y}, \frac{\partial \varphi}{\partial z}\right) .
$$

In the resultant antiplane deformation all the field variables are independent of $z$. The components of the phonon and phason stresses and electric displacement are therefore given by Eqs. (5) and (6) as

$$
\begin{gathered}
\sigma_{y z}=2 c_{44} \varepsilon_{y z}+R w_{z y}-e_{15} E_{y}, \\
\sigma_{x z}=2 c_{44} \varepsilon_{x z}+R w_{z x}-e_{15} E_{x}, \\
H_{z y}=2 R \varepsilon_{y z}+K w_{z y}-e_{15}^{\prime} E_{y}, \\
H_{z x}=2 R \varepsilon_{x z}+K w_{z x}-e_{15}^{\prime} E_{x}, \\
D_{y}=2 e_{15} \varepsilon_{y z}+e_{15}^{\prime} w_{z y}+\varepsilon_{11} E_{y}, \\
D_{x}=2 e_{15} \varepsilon_{x z}+e_{15}^{\prime} w_{z x}+\varepsilon_{11} E_{x},
\end{gathered}
$$

and the corresponding relations between the components $u_{z}$ and $w_{z}$ of the phonon and phason displacements and the non-zero phonon and phason strain components are

$$
\begin{aligned}
& \varepsilon_{x z}=\frac{1}{2} \frac{\partial u_{z}}{\partial x}, \quad \varepsilon_{y z}=\frac{1}{2} \frac{\partial u_{z}}{\partial y}, \\
& w_{z x}=\frac{\partial w_{z}}{\partial x}, \quad w_{z y}=\frac{\partial w_{z}}{\partial y},
\end{aligned}
$$

where here, and subsequently, to induce brevity of the presentation, the abbreviations $R$ and $K$ are used for the constants $R_{3}$ and $K_{2}$. 
The mode III antiplane deformation is created by the application to the piezoelectric quasicrystal of nonuniform phonon, phason and electrical loads, $\mathcal{T}(\xi), \mathcal{H}(\xi)$ and $\mathcal{D}(\xi)$, which are periodic and symmetrical within the intervals $S_{t}$ such that at infinity

$$
\begin{aligned}
\sigma_{y z}(\xi, y) & \rightarrow \mathcal{T}(\xi), \quad H_{z y}(\xi, y) \rightarrow \mathcal{H}(\xi), \\
D_{y}(\xi, y) & \rightarrow \mathcal{D}(\xi),
\end{aligned}
$$

where $\mathcal{T}(-\xi)=\mathcal{T}(\xi)=\mathcal{T}(\xi+2 n h)=\mathcal{T}(-\xi+2 n h)$, $\mathcal{H}(-\xi)=\mathcal{H}(\xi)=\mathcal{H}(\xi+2 n h)=\mathcal{H}(-\xi+2 n h)$ and $\mathcal{D}(-\xi)=\mathcal{D}(\xi)=\mathcal{D}(\xi+2 n h)=\mathcal{D}(-\xi+2 n h)$ for $n=0$, $\pm 1, \pm 2 \ldots$

Analyses that are analogous to those presented here could be developed as desired by a reader, if instead any other preferred specifications of three of the phonon, phason and electrical components $\sigma_{y z}, \varepsilon_{y z}, H_{z y}, w_{z y}, D_{y}$, or $E_{y}$ are preferable.

There is a continuing upsurge in the development of important, innovative, technological applications of quasicrystals, but it is of interest to note that there is not yet a consensus upon the ways in which physically the phason loads should be imposed. Typically, for example, it was observed by Sladek et al. [27] that "... a physical interpretation on these phason forces is still missing..." and by $\mathrm{Li}[28]$ that "Although no experiments have re- ported yet on how to impose the phason loads, within the theory of elasticity of QC..., however, traction in the phason field must exist on the boundary of a $\mathrm{QC}$, from a theoretical point of view."

\section{Screw dislocation moving in a piezoelectric quasicrystal}

As an antecedent, it is appropriate to briefly outline the fundamental concept of a moving "piezoelectric quasicrystal screw dislocation" upon which the foundations of the present analysis depend. This is an extension of the original concept of a dislocation in a classical elastic solid in which a more comprehensive Burgers vector is introduced with slip-plane discontinuities of magnitudes $b, d$ and $b_{4}$, respectively, in the components $u_{z}, w_{z}$ and $\phi$ of the phonon displacement, the phason displacement and the electric potential.

For such a straight dislocation at the origin that is parallel to the $z$-axis and moving at a speed $v$ along the $x$-axis in a one-dimensional hexagonal piezoelectric quasicrystal with point group $6 \mathrm{~mm}$, Wang and Pan [12] derived explicit results for its field components. After conveniently renaming and algebraically regrouping some of the moduli of the material, these become

$$
\begin{aligned}
& \left.u_{z}^{\mathrm{III}}(\xi, y)=\frac{1}{2 \pi\left(\alpha^{2}+\bar{R}^{2}\right)}\left\{b\left[\alpha^{2} \tan ^{-1}\left(\frac{\beta_{1} y}{\xi}\right)+\bar{R}^{2} \tan ^{-1}\left(\frac{\beta_{2} y}{\xi}\right)\right]+d \alpha \bar{R}\left[\tan ^{-1}\left(\frac{\beta_{1} y}{\xi}\right)-\tan ^{-1}\left(\frac{\beta_{2} y}{\xi}\right)\right]\right\}, \quad(18)\right] \\
& w_{z}^{\mathrm{III}}(\xi, y)=\frac{1}{2 \pi\left(\alpha^{2}+\bar{R}^{2}\right)}\left\{b \alpha \bar{R}\left[\tan ^{-1}\left(\frac{\beta_{1} y}{\xi}\right)-\tan ^{-1}\left(\frac{\beta_{2} y}{\xi}\right)\right]+d\left[\bar{R}^{2} \tan ^{-1}\left(\frac{\beta_{1} y}{\xi}\right)+\alpha^{2} \tan ^{-1}\left(\frac{\beta_{2} y}{\xi}\right)\right]\right\}, \quad(19) \\
& \varphi^{\mathrm{III}}(\xi, y)=\frac{1}{2 \pi}\left\{b\left[\frac{1}{\varepsilon_{11}\left(\alpha^{2}+\bar{R}^{2}\right)}\left(\alpha\left(e_{15} \alpha+e_{15}^{\prime} \bar{R}\right) \tan ^{-1}\left(\frac{\beta_{1} y}{\xi}\right)-\bar{R}\left(e_{15}^{\prime} \alpha-e_{15} \bar{R}\right) \tan ^{-1}\left(\frac{\beta_{2} y}{\xi}\right)\right)-\frac{e_{15}}{\varepsilon_{11}} \tan ^{-1}\left(\frac{y}{\xi}\right)\right]\right. \\
& \quad+d\left[\frac{1}{\varepsilon_{11}\left(\alpha^{2}+\bar{R}^{2}\right)}\left(\bar{R}\left(e_{15} \alpha+e_{15}^{\prime} \bar{R}\right) \tan ^{-1}\left(\frac{\beta_{1} y}{\xi}\right)+\alpha\left(e_{15}^{\prime} \alpha-e_{15} \bar{R}\right) \tan ^{-1}\left(\frac{\beta_{2} y}{\xi}\right)\right)-\frac{e_{15}^{\prime}}{\varepsilon_{11}} \tan ^{-1}\left(\frac{y}{\xi}\right)\right] \\
& \left.\quad+b_{4} \tan ^{-1}\left(\frac{y}{\xi}\right)\right\},
\end{aligned}
$$

with the III superscript indicating throughout their association to the mode III antiplane deformation.

For brevity it is convenient to define

$$
\alpha=\left(\bar{c}_{44}-\bar{K}+\sqrt{\left(\bar{c}_{44}-\bar{K}\right)^{2}+4 \bar{R}^{2}}\right) / 2
$$

in terms of the piezoelectrically stiffened phonon and phason elastic moduli, $\bar{c}_{44}$ and $\bar{K}$, and the phononphason coupling elastic constant, $\bar{R}$, given by

$$
\begin{aligned}
& \bar{c}_{44}=c_{44}+\frac{e_{15}^{2}}{\varepsilon_{11}}, \\
& \bar{K}=K+\frac{e_{15}^{\prime 2}}{\varepsilon_{11}}, \\
& \bar{R}=R+\frac{e_{15} e_{15}^{\prime}}{\varepsilon_{11}},
\end{aligned}
$$

and

$$
\beta_{i}=\sqrt{1-\frac{v^{2}}{s_{i}^{2}}} \text { for } i=1 \text { and } 2,
$$

where the piezoelectrically stiffened wave speeds, $s_{1}$ and $s_{2}$, are given under antiplane shear conditions by

$$
s_{i}=\sqrt{\varepsilon_{i} / \rho},
$$

with

$$
\begin{aligned}
& \varepsilon_{1}=\left[\bar{c}_{44}+\bar{K}+\sqrt{\left(\bar{c}_{44}-\bar{K}\right)^{2}+4 \bar{R}^{2}}\right] / 2, \\
& \varepsilon_{2}=\left[\bar{c}_{44}+\bar{K}-\sqrt{\left(\bar{c}_{44}-\bar{K}\right)^{2}+4 \bar{R}^{2}}\right] / 2 .
\end{aligned}
$$

Substitution of the expressions (18)-(20) into the constitutive equations (12)-(14), using the results (15), (16) and (11) yields the non-zero phonon and phason stresses and electric displacement components to be 


$$
\begin{aligned}
& \sigma_{x z}^{\mathrm{III}}(\xi, y)=-\frac{y}{2 \pi}\left\{b\left[\frac{1}{\left(\alpha^{2}+\bar{R}^{2}\right)}\left(\frac{\beta_{1} \alpha\left(\bar{c}_{44} \alpha+\bar{R}^{2}\right)}{\xi^{2}+\beta_{1}^{2} y^{2}}+\frac{\beta_{2} \bar{R}^{2}\left(\bar{c}_{44}-\alpha\right)}{\xi^{2}+\beta_{2}^{2} y^{2}}\right)-\frac{e_{15}^{2}}{\varepsilon_{11}\left(\xi^{2}+y^{2}\right)}\right]\right. \\
& \left.+d\left[\frac{\bar{R}}{\left(\alpha^{2}+\bar{R}^{2}\right)}\left(\frac{\beta_{1}\left(\bar{c}_{44} \alpha+\bar{R}^{2}\right)}{\xi^{2}+\beta_{1}^{2} y^{2}}-\frac{\beta_{2} \alpha\left(\bar{c}_{44}-\alpha\right)}{\xi^{2}+\beta_{2}^{2} y^{2}}\right)-\frac{e_{15} e_{15}^{\prime}}{\varepsilon_{11}\left(\xi^{2}+y^{2}\right)}\right]+b_{4} \frac{e_{15}}{\xi^{2}+y^{2}}\right\}, \\
& \sigma_{y z}^{\mathrm{III}}(\xi, y)=\frac{\xi}{2 \pi}\left\{b\left[\frac{1}{\left(\alpha^{2}+\bar{R}^{2}\right)}\left(\frac{\beta_{1} \alpha\left(\bar{c}_{44} \alpha+\bar{R}^{2}\right)}{\xi^{2}+\beta_{1}^{2} y^{2}}+\frac{\beta_{2} \bar{R}^{2}\left(\bar{c}_{44}-\alpha\right)}{\xi^{2}+\beta_{2}^{2} y^{2}}\right)-\frac{e_{15}^{2}}{\varepsilon_{11}\left(\xi^{2}+y^{2}\right)}\right]\right. \\
& \left.+d\left[\frac{\bar{R}}{\left(\alpha^{2}+\bar{R}^{2}\right)}\left(\frac{\beta_{1}\left(\bar{c}_{44} \alpha+\bar{R}^{2}\right)}{\xi^{2}+\beta_{1}^{2} y^{2}}-\frac{\beta_{2} \alpha\left(\bar{c}_{44}-\alpha\right)}{\xi^{2}+\beta_{2}^{2} y^{2}}\right)-\frac{e_{15} e_{15}^{\prime}}{\varepsilon_{11}\left(\xi^{2}+y^{2}\right)}\right]+b_{4} \frac{e_{15}}{\xi^{2}+y^{2}}\right\}, \\
& H_{z x}^{\mathrm{III}}(\xi, y)=-\frac{y}{2 \pi}\left\{b\left[\frac{\bar{R}}{\left(\alpha^{2}+\bar{R}^{2}\right)}\left(\frac{\beta_{1} \alpha(\alpha+\bar{K})}{\xi^{2}+\beta_{1}^{2} y^{2}}-\frac{\beta_{2}\left(\alpha \bar{K}-\bar{R}^{2}\right)}{\xi^{2}+\beta_{2}^{2} y^{2}}\right)-\frac{e_{15} e_{15}^{\prime}}{\varepsilon_{11}\left(\xi^{2}+y^{2}\right)}\right]\right. \\
& \left.+d\left[\frac{1}{\left(\alpha^{2}+\bar{R}^{2}\right)}\left(\frac{\beta_{1} \bar{R}^{2}(\alpha+\bar{K})}{\xi^{2}+\beta_{1}^{2} y^{2}}+\frac{\beta_{2} \alpha\left(\alpha \bar{K}-\bar{R}^{2}\right)}{\xi^{2}+\beta_{2}^{2} y^{2}}\right)-\frac{e_{15}^{\prime 2}}{\varepsilon_{11}\left(\xi^{2}+y^{2}\right)}\right]+b_{4} \frac{e_{15}^{\prime}}{\xi^{2}+y^{2}}\right\}, \\
& H_{z y}^{\mathrm{III}}(\xi, y)=\frac{\xi}{2 \pi}\left\{b\left[\frac{\bar{R}}{\left(\alpha^{2}+\bar{R}^{2}\right)}\left(\frac{\beta_{1} \alpha(\alpha+\bar{K})}{\xi^{2}+\beta_{1}^{2} y^{2}}-\frac{\beta_{2}\left(\alpha \bar{K}-\bar{R}^{2}\right)}{\xi^{2}+\beta_{2}^{2} y^{2}}\right)-\frac{e_{15} e_{15}^{\prime}}{\varepsilon_{11}\left(\xi^{2}+y^{2}\right)}\right]\right. \\
& \left.+d\left[\frac{1}{\left(\alpha^{2}+\bar{R}^{2}\right)}\left(\frac{\beta_{1} \bar{R}^{2}(\alpha+\bar{K})}{\xi^{2}+\beta_{1}^{2} y^{2}}+\frac{\beta_{2} \alpha\left(\alpha \bar{K}-\bar{R}^{2}\right)}{\xi^{2}+\beta_{2}^{2} y^{2}}\right)-\frac{e_{15}^{\prime 2}}{\varepsilon_{11}\left(\xi^{2}+y^{2}\right)}\right]+b_{4} \frac{e_{15}^{\prime}}{\xi^{2}+y^{2}}\right\},
\end{aligned}
$$

$$
\begin{aligned}
& D_{x}^{\mathrm{III}}(\xi, y)=-\frac{y}{2 \pi} \frac{b e_{15}+d e_{15}^{\prime}-b_{4} \varepsilon_{11}}{\xi^{2}+y^{2}}, \\
& D_{y}^{\mathrm{III}}(\xi, y)=\frac{\xi}{2 \pi} \frac{b e_{15}+d e_{15}^{\prime}-b_{4} \varepsilon_{11}}{\xi^{2}+y^{2}} .
\end{aligned}
$$

Correspondingly Eqs. (18)-(20) together with Eqs. (11), (15) and (16) enable the analogous expressions for the phonon and phason strain and electric field components to be derived if desired.

\section{Moving row of cracks analysis using the extended dislocation layers method}

The crucial feature which originally lead to the exploitation of the "dislocation layer method" for isotropic elastic solids is that a loaded strip crack can be mod-

elled as an appropriate array of elastic dislocations, as discussed conveniently by Bilby and Eshelby [29] and Lardner [30], for example. This powerful technique is extended to instigate the present study of a row of moving cracks in piezoelectric quasicrystals by spreading a suitable distribution of moving piezoelectric quasicrystal screw dislocations.

The screws are positive to the right of each crack and negative to the left. The density functions, $f(\xi), g(\xi)$, and $j(\xi)$, of the discontinuities in the phonon and phason displacements components and the electric potential, respectively, are thus necessarily all odd functions of $\xi$.

The relevant corresponding components of the phonon and phason stresses and electric displacement created at a point on the $\xi$-axis by the array are given by Eqs. (27), (29) and (31) as

$$
\begin{aligned}
& \sigma_{y z}^{\mathrm{III}}(\xi, 0)=\frac{b}{2 \pi}\left[\left(\frac{\beta_{1} \alpha\left(\bar{c}_{44} \alpha+\bar{R}^{2}\right)+\beta_{2} \bar{R}^{2}\left(\bar{c}_{44}-\alpha\right)}{\alpha^{2}+\bar{R}^{2}}\right)-\frac{e_{15}^{2}}{\varepsilon_{11}}\right] \int_{-\infty}^{\infty} \frac{f\left(\xi^{\prime}\right)}{\xi-\xi^{\prime}} \mathrm{d} \xi^{\prime} \\
& +\frac{d}{2 \pi}\left[\bar{R}\left(\frac{\beta_{1}\left(\bar{c}_{44} \alpha+\bar{R}^{2}\right)-\beta_{2} \alpha\left(\bar{c}_{44}-\alpha\right)}{\alpha^{2}+\bar{R}^{2}}\right)-\frac{e_{15} e_{15}^{\prime}}{\varepsilon_{11}}\right] \int_{-\infty}^{\infty} \frac{g\left(\xi^{\prime}\right)}{\xi-\xi^{\prime}} \mathrm{d} \xi^{\prime}+\frac{b_{4} e_{15}}{2 \pi} \int_{-\infty}^{\infty} \frac{j\left(\xi^{\prime}\right)}{\xi-\xi^{\prime}} \mathrm{d} \xi^{\prime}, \\
& H_{z y}^{\mathrm{III}}(\xi, 0)=\frac{b}{2 \pi}\left[\bar{R}\left(\frac{\beta_{1} \alpha(\alpha+\bar{K})-\beta_{2}\left(\alpha \bar{K}-\bar{R}^{2}\right)}{\alpha^{2}+\bar{R}^{2}}\right)-\frac{e_{15} e_{15}^{\prime}}{\varepsilon_{11}}\right] \int_{-\infty}^{\infty} \frac{f\left(\xi^{\prime}\right)}{\xi-\xi^{\prime}} \mathrm{d} \xi^{\prime} \\
& +\frac{d}{2 \pi}\left[\left(\frac{\beta_{1} \bar{R}^{2}(\alpha+\bar{K})+\beta_{2} \alpha\left(\alpha \bar{K}-\bar{R}^{2}\right)}{\alpha^{2}+\bar{R}^{2}}\right)-\frac{e_{15}^{\prime}{ }^{2}}{\varepsilon_{11}}\right] \int_{-\infty}^{\infty} \frac{g\left(\xi^{\prime}\right)}{\xi-\xi^{\prime}} \mathrm{d} \xi^{\prime}+\frac{b_{4} e_{15}^{\prime}}{2 \pi} \int_{-\infty}^{\infty} \frac{j\left(\xi^{\prime}\right)}{\xi-\xi^{\prime}} \mathrm{d} \xi^{\prime},
\end{aligned}
$$




$$
\begin{aligned}
& D_{y}^{\mathrm{III}}(\xi, 0)=\frac{b e_{15}}{2 \pi} \int_{-\infty}^{\infty} \frac{f\left(\xi^{\prime}\right)}{\xi-\xi^{\prime}} \mathrm{d} \xi^{\prime}+\frac{d e_{15}^{\prime}}{2 \pi} \int_{-\infty}^{\infty} \frac{g\left(\xi^{\prime}\right)}{\xi-\xi^{\prime}} \mathrm{d} \xi^{\prime} \\
&-\frac{b_{4} \varepsilon_{11}}{2 \pi} \int_{-\infty}^{\infty} \frac{j\left(\xi^{\prime}\right)}{\xi-\xi^{\prime}} \mathrm{d} \xi^{\prime} .
\end{aligned}
$$

The improper integrals in Eqs. (32)-(34) need to be interpreted using their Cauchy principal values to accord with the Plemelj formulae.

The equilibrium equations which must be satisfied by the dislocation array to fulfil the imposed boundary conditions (17) are that

$$
\begin{aligned}
& \sigma_{y z}^{\mathrm{III}}(\xi, 0)=-\mathcal{T}(\xi), \\
& H_{z y}^{\mathrm{III}}(\xi, 0)=-\mathcal{H}(\xi), \\
& D_{y}^{\mathrm{III}}(\xi, 0)=-\mathcal{D}(\xi) \text { in } R_{t} .
\end{aligned}
$$

Substitution of the representations (32)-(34) into these yields a system of three simultaneous equations whose solutions lead to three singular integral equations for the determination of the densities $f(\xi), g(\xi)$ and $j(\xi)$. After much detailed algebraic simplification and manipulation, they can be succinctly expressed as

$$
\begin{aligned}
& \int_{-\infty}^{\infty} \frac{f\left(\xi^{\prime}\right)}{\xi-\xi^{\prime}} \mathrm{d} \xi^{\prime}=-\frac{2 \pi}{b \beta_{1} \beta_{2} \varepsilon_{11}\left(\bar{c}_{44} \bar{K}-\bar{R}^{2}\right)\left(\alpha^{2}+\bar{R}^{2}\right)} \\
& \times\left\{\varepsilon_{11}\left(\beta_{1} \bar{R}^{2}(\alpha+\bar{K})+\beta_{2} \alpha\left(\alpha \bar{K}-\bar{R}^{2}\right)\right) \mathcal{T}(\xi)-\varepsilon_{11} \bar{R}\left(\beta_{1}\left(\bar{c}_{44} \alpha+\bar{R}^{2}\right)-\beta_{2} \alpha\left(\bar{c}_{44}-\alpha\right)\right) \mathcal{H}(\xi)\right. \\
& \left.-\left[e_{15}^{\prime} \bar{R}\left(\beta_{1}\left(\bar{c}_{44} \alpha+\bar{R}^{2}\right)-\beta_{2} \alpha\left(\bar{c}_{44}-\alpha\right)\right)-e_{15}\left(\beta_{1} \bar{R}^{2}(\alpha+\bar{K})+\beta_{2} \alpha\left(\alpha \bar{K}-\bar{R}^{2}\right)\right)\right] \mathcal{D}(\xi)\right\}, \\
& \int_{-\infty}^{\infty} \frac{g\left(\xi^{\prime}\right)}{\xi-\xi^{\prime}} \mathrm{d} \xi^{\prime}=-\frac{2 \pi}{d \beta_{1} \beta_{2} \varepsilon_{11}\left(\bar{c}_{44} \bar{K}-\bar{R}^{2}\right)\left(\alpha^{2}+\bar{R}^{2}\right)} \\
& \times\left\{-\varepsilon_{11} \bar{R}\left(\beta_{1} \alpha(\alpha+\bar{K})-\beta_{2}\left(\alpha \bar{K}-\bar{R}^{2}\right)\right) \mathcal{T}(\xi)+\varepsilon_{11}\left(\beta_{1} \alpha\left(\bar{c}_{44} \alpha+\bar{R}^{2}\right)+\beta_{2} \bar{R}^{2}\left(\bar{c}_{44}-\alpha\right)\right) \mathcal{H}(\xi)\right. \\
& \left.+\left[e_{15}^{\prime}\left(\beta_{1} \alpha\left(\bar{c}_{44} \alpha+\bar{R}^{2}\right)+\beta_{2} \bar{R}^{2}\left(\bar{c}_{44}-\alpha\right)\right)-e_{15} \bar{R}\left(\beta_{1} \alpha(\alpha+\bar{K})-\beta_{2}\left(\alpha \bar{K}-\bar{R}^{2}\right)\right)\right] \mathcal{D}(\xi)\right\}, \\
& \int_{-\infty}^{\infty} \frac{j\left(\xi^{\prime}\right)}{\xi-\xi^{\prime}} \mathrm{d} \xi^{\prime}=-\frac{2 \pi}{b_{4} \beta_{1} \beta_{2} \varepsilon_{11}\left(\bar{c}_{44} \bar{K}-\bar{R}^{2}\right)\left(\alpha^{2}+\bar{R}^{2}\right)} \\
& \times\left\{-\left[e_{15}^{\prime} \bar{R}\left(\beta_{1} \alpha(\alpha+\bar{K})-\beta_{2}\left(\alpha \bar{K}-\bar{R}^{2}\right)\right)-e_{15}\left(\beta_{1} \bar{R}^{2}(\alpha+\bar{K})+\beta_{2} \alpha\left(\alpha \bar{K}-\bar{R}^{2}\right)\right)\right] \mathcal{T}(\xi)\right. \\
& +\left[e_{15}^{\prime}\left(\beta_{1} \alpha\left(\bar{c}_{44} \alpha+\bar{R}^{2}\right)+\beta_{2} \bar{R}^{2}\left(\bar{c}_{44}-\alpha\right)\right)-e_{15} \bar{R}\left(\beta_{1}\left(\bar{c}_{44} \alpha+\bar{R}^{2}\right)-\beta_{2} \alpha\left(\bar{c}_{44}-\alpha\right)\right)\right] \mathcal{H}(\xi) \\
& -\left[\beta_{1} \beta_{2}\left(\bar{c}_{44} \bar{K}-\bar{R}^{2}\right)\left(\alpha^{2}+\bar{R}^{2}\right)-\frac{e_{15}^{2}}{\varepsilon_{11}}\left(\beta_{1} \bar{R}^{2}(\alpha+\bar{K})+\beta_{2} \alpha\left(\alpha \bar{K}-\bar{R}^{2}\right)\right)\right. \\
& -\frac{e_{15}^{\prime 2}}{\varepsilon_{11}}\left(\beta_{1} \alpha\left(\bar{c}_{44} \alpha+\bar{R}^{2}\right)+\beta_{2} \bar{R}^{2}\left(\bar{c}_{44}-\alpha\right)\right)+\frac{e_{15} e_{15}^{\prime}}{\varepsilon_{11}} \bar{R}\left(\beta_{1}\left(\alpha(\alpha+\bar{K})+\left(\bar{c}_{44} \alpha+\bar{R}^{2}\right)\right)\right. \\
& \left.\left.\left.-\beta_{2}\left(\alpha\left(\bar{c}_{44}-\alpha\right)+\left(\alpha \bar{K}-\bar{R}^{2}\right)\right)\right)\right] \mathcal{D}(\xi)\right\} .
\end{aligned}
$$

But it is convenient to now express the infinite integrals which appear in Eqs. (36)-(38) as sums of the individual contributions from each of the cracks in the row using the identity

$$
\sum_{n=1}^{\infty} \frac{1}{z^{2}-n^{2}}=-\frac{1}{2 z^{2}}+\frac{\pi}{2 z} \cot \pi z
$$

and recalling that the density functions are odd and periodic. The left-hand side of Eq. (36), for example, is then found (see, for example, Leibfried [31]) to have the alternative representation

$$
\frac{\pi}{2 h} \int_{-a}^{a} \frac{\cos \left(\pi \xi^{\prime} / 2 h\right)}{\sin (\pi \xi / 2 h)-\sin \left(\pi \xi^{\prime} / 2 h\right)} f\left(\xi^{\prime}\right) \mathrm{d} \xi^{\prime},
$$

which, by introducing new variables as

$$
\begin{aligned}
& \xi_{1}=\sin (\pi \xi / 2 h), \\
& a_{1}=\sin (\pi a / 2 h), \\
& \xi_{1}^{\prime}=\sin \left(\pi \xi^{\prime} / 2 h\right),
\end{aligned}
$$

transforms the integral Eq. (36) for $f(\xi)$ into the expedient form 


$$
\begin{aligned}
& \int_{-a_{1}}^{a_{1}} \frac{f_{1}\left(\xi_{1}^{\prime}\right)}{\xi_{1}-\xi_{1}^{\prime}} \mathrm{d} \xi_{1}^{\prime}=-\frac{2 \pi}{b \beta_{1} \beta_{2} \varepsilon_{11}\left(\bar{c}_{44} \bar{K}-\bar{R}^{2}\right)\left(\alpha^{2}+\bar{R}^{2}\right)} \\
& \quad \times\left\{\varepsilon_{11}\left(\beta_{1} \bar{R}^{2}(\alpha+\bar{K})+\beta_{2} \alpha\left(\alpha \bar{K}-\bar{R}^{2}\right)\right) \mathcal{T}(\xi)-\varepsilon_{11} \bar{R}\left(\beta_{1}\left(\bar{c}_{44} \alpha+\bar{R}^{2}\right)-\beta_{2} \alpha\left(\bar{c}_{44}-\alpha\right)\right) \mathcal{H}(\xi)\right. \\
& \left.\quad-\left[e_{15}^{\prime} \bar{R}\left(\beta_{1}\left(\bar{c}_{44} \alpha+\bar{R}^{2}\right)-\beta_{2} \alpha\left(\bar{c}_{44}-\alpha\right)\right)-e_{15}\left(\beta_{1} \bar{R}^{2}(\alpha+\bar{K})+\beta_{2} \alpha\left(\alpha \bar{K}-\bar{R}^{2}\right)\right)\right] \mathcal{D}(\xi)\right\}
\end{aligned}
$$

for $\left|\xi_{1}\right|<a_{1}$,

with $f_{1}\left(\xi_{1}\right)=f_{1}(\sin (\pi \xi / 2 h)) \equiv f(\xi)$. Its appropriate solution can be shown by adapting traditional methods, as discussed by Muskhelishvili [32], and Gakhov [33], for example, to be

$$
\begin{aligned}
& f_{1}\left(\xi_{1}\right)=-\frac{2}{\pi b \beta_{1} \beta_{2} \varepsilon_{11}\left(\bar{c}_{44} \bar{K}-\bar{R}^{2}\right)\left(\alpha^{2}+\bar{R}^{2}\right)} \frac{1}{\left(a_{1}^{2}-\xi_{1}^{2}\right)^{\frac{1}{2}}} \int_{-a_{1}}^{a_{1}} \frac{\left(a_{1}^{2}-\xi_{1}^{\prime 2}\right)^{\frac{1}{2}}}{\xi_{1}^{\prime}-\xi_{1}} \\
& \quad \times\left\{\varepsilon_{11}\left(\beta_{1} \bar{R}^{2}(\alpha+\bar{K})+\beta_{2} \alpha\left(\alpha \bar{K}-\bar{R}^{2}\right)\right) \mathcal{T}\left(\xi_{1}^{\prime}\right)-\varepsilon_{11} \bar{R}\left(\beta_{1}\left(\bar{c}_{44} \alpha+\bar{R}^{2}\right)-\beta_{2} \alpha\left(\bar{c}_{44}-\alpha\right)\right) \mathcal{H}\left(\xi_{1}^{\prime}\right)\right. \\
& \left.\quad-\left[e_{15}^{\prime} \bar{R}\left(\beta_{1}\left(\bar{c}_{44} \alpha+\bar{R}^{2}\right)-\beta_{2} \alpha\left(\bar{c}_{44}-\alpha\right)\right)-e_{15}\left(\beta_{1} \bar{R}^{2}(\alpha+\bar{K})+\beta_{2} \alpha\left(\alpha \bar{K}-\bar{R}^{2}\right)\right)\right] \mathcal{D}\left(\xi_{1}^{\prime}\right)\right\} \mathrm{d} \xi_{1}^{\prime} .
\end{aligned}
$$

Analogously it can be deduced that the phason and electric densities which satisfy Eqs. (37) and (38) are

$$
\begin{aligned}
& g_{1}\left(\xi_{1}\right)=-\frac{2}{\pi d \beta_{1} \beta_{2} \varepsilon_{11}\left(\bar{c}_{44} \bar{K}-\bar{R}^{2}\right)\left(\alpha^{2}+\bar{R}^{2}\right)} \frac{1}{\left(a_{1}^{2}-\xi_{1}^{2}\right)^{\frac{1}{2}}} \int_{-a_{1}}^{a_{1}} \frac{\left(a_{1}^{2}-\xi_{1}^{\prime 2}\right)^{\frac{1}{2}}}{\xi_{1}^{\prime}-\xi_{1}} \\
& \times\left\{-\varepsilon_{11} \bar{R}\left(\beta_{1} \alpha(\alpha+\bar{K})-\beta_{2}\left(\alpha \bar{K}-\bar{R}^{2}\right)\right) \mathcal{T}\left(\xi_{1}^{\prime}\right)+\varepsilon_{11}\left(\beta_{1} \alpha\left(\bar{c}_{44} \alpha+\bar{R}^{2}\right)+\beta_{2} \bar{R}^{2}\left(\bar{c}_{44}-\alpha\right)\right) \mathcal{H}\left(\xi_{1}^{\prime}\right)\right. \\
& \left.+\left[e_{15}^{\prime}\left(\beta_{1} \alpha\left(\bar{c}_{44} \alpha+\bar{R}^{2}\right)+\beta_{2} \bar{R}^{2}\left(\bar{c}_{44}-\alpha\right)\right)-e_{15} \bar{R}\left(\beta_{1} \alpha(\alpha+\bar{K})-\beta_{2}\left(\alpha \bar{K}-\bar{R}^{2}\right)\right)\right] \mathcal{D}\left(\xi_{1}^{\prime}\right)\right\} \mathrm{d} \xi_{1}^{\prime}, \\
& j_{1}\left(\xi_{1}\right)=-\frac{2}{\pi b_{4} \beta_{1} \beta_{2} \varepsilon_{11}\left(\bar{c}_{44} \bar{K}-\bar{R}^{2}\right)\left(\alpha^{2}+\bar{R}^{2}\right)} \frac{1}{\left(a_{1}^{2}-\xi_{1}^{2}\right)^{\frac{1}{2}}} \int_{-a_{1}}^{a_{1}} \frac{\left(a_{1}^{2}-\xi_{1}^{\prime 2}\right)^{\frac{1}{2}}}{\xi_{1}^{\prime}-\xi_{1}} \\
& \times\left\{-\left[e_{15}^{\prime} \bar{R}\left(\beta_{1} \alpha(\alpha+\bar{K})-\beta_{2}\left(\alpha \bar{K}-\bar{R}^{2}\right)\right)-e_{15}\left(\beta_{1} \bar{R}^{2}(\alpha+\bar{K})+\beta_{2} \alpha\left(\alpha \bar{K}-\bar{R}^{2}\right)\right)\right] \mathcal{T}\left(\xi_{1}^{\prime}\right)\right. \\
& +\left[e_{15}^{\prime}\left(\beta_{1} \alpha\left(\bar{c}_{44} \alpha+\bar{R}^{2}\right)+\beta_{2} \bar{R}^{2}\left(\bar{c}_{44}-\alpha\right)\right)-e_{15} \bar{R}\left(\beta_{1}\left(\bar{c}_{44} \alpha+\bar{R}^{2}\right)-\beta_{2} \alpha\left(\bar{c}_{44}-\alpha\right)\right)\right] \mathcal{H}\left(\xi_{1}^{\prime}\right) \\
& -\left[\beta_{1} \beta_{2}\left(\bar{c}_{44} \bar{K}-\bar{R}^{2}\right)\left(\alpha^{2}+\bar{R}^{2}\right)-\frac{e_{15}^{2}}{\varepsilon_{11}}\left(\beta_{1} \bar{R}^{2}(\alpha+\bar{K})+\beta_{2} \alpha\left(\alpha \bar{K}-\bar{R}^{2}\right)\right)\right. \\
& -\frac{e_{15}^{\prime 2}}{\varepsilon_{11}}\left(\beta_{1} \alpha\left(\bar{c}_{44} \alpha+\bar{R}^{2}\right)+\beta_{2} \bar{R}^{2}\left(\bar{c}_{44}-\alpha\right)\right)+\frac{e_{15} e_{15}^{\prime}}{\varepsilon_{11}} \bar{R}\left(\beta_{1}\left(\alpha(\alpha+\bar{K})+\left(\bar{c}_{44} \alpha+\bar{R}^{2}\right)\right)\right. \\
& \left.\left.\left.-\beta_{2}\left(\alpha\left(\bar{c}_{44}-\alpha\right)+\left(\alpha \bar{K}-\bar{R}^{2}\right)\right)\right)\right] \mathcal{D}\left(\xi_{1}^{\prime}\right)\right\} \mathrm{d} \xi_{1}^{\prime},
\end{aligned}
$$

with $g_{1}\left(\xi_{1}\right)=g_{1}(\sin (\pi \xi / 2 h)) \equiv g(\xi)$,

$$
j_{1}\left(\xi_{1}\right)=j_{1}(\sin (\pi \xi / 2 h)) \equiv j(\xi) .
$$

It is observed that, in general, all the densities are dependent upon the applied loads $\mathcal{T}(\xi), \mathcal{H}(\xi)$ and $\mathcal{D}(\xi)$, the geometric constants $a$ and $h$, the material constants of the piezoelectric quasicrystal and also the speed of the cracks.

With these expressions for the appropriate densities $f(\xi), g(\xi)$ and $j(\xi)$ now available, explicit representations can be derived from Eqs. (26)-(31) and (43)-(45) for the phonon and phason stress and electric fields' components that are of interest.

For example, as a demonstration of the type of analyses needed, it follows from Eqs. (17), (27) and (26) that: 


$$
\begin{aligned}
& \sigma_{y z}(\xi, y)=\mathcal{T}(\xi) \\
& +\frac{b}{2 \pi} \int_{-\infty}^{\infty}\left(\xi-\xi^{\prime \prime}\right)\left[\frac{1}{\left(\alpha^{2}+\bar{R}^{2}\right)}\left(\frac{\beta_{1} \alpha\left(\bar{c}_{44} \alpha+\bar{R}^{2}\right)}{\left(\xi-\xi^{\prime \prime}\right)^{2}+\beta_{1}^{2} y^{2}}+\frac{\beta_{2} \bar{R}^{2}\left(\bar{c}_{44}-\alpha\right)}{\left(\xi-\xi^{\prime \prime}\right)^{2}+\beta_{2}^{2} y^{2}}\right)-\frac{e_{15}^{2}}{\varepsilon_{11}\left(\left(\xi-\xi^{\prime \prime}\right)^{2}+y^{2}\right)}\right] f\left(\xi^{\prime \prime}\right) \mathrm{d} \xi^{\prime \prime} \\
& +\frac{d}{2 \pi} \int_{-\infty}^{\infty}\left(\xi-\xi^{\prime \prime}\right)\left[\frac{\bar{R}}{\left(\alpha^{2}+\bar{R}^{2}\right)}\left(\frac{\beta_{1}\left(\bar{c}_{44} \alpha+\bar{R}^{2}\right)}{\left(\xi-\xi^{\prime \prime}\right)^{2}+\beta_{1}^{2} y^{2}}-\frac{\beta_{2} \alpha\left(\bar{c}_{44}-\alpha\right)}{\left(\xi-\xi^{\prime \prime}\right)^{2}+\beta_{2}^{2} y^{2}}\right)-\frac{e_{15} e_{15}^{\prime}}{\varepsilon_{11}\left(\left(\xi-\xi^{\prime \prime}\right)^{2}+y^{2}\right)}\right] g\left(\xi^{\prime \prime}\right) \mathrm{d} \xi^{\prime \prime} \\
& +\frac{b_{4}}{2 \pi} \int_{-\infty}^{\infty}\left(\xi-\xi^{\prime \prime}\right) \frac{e_{15}}{\left(\xi-\xi^{\prime \prime}\right)^{2}+y^{2}} j\left(\xi^{\prime \prime}\right) \mathrm{d} \xi^{\prime \prime}, \\
& \sigma_{x z}(\xi, y)=-\frac{b y}{2 \pi} \int_{-\infty}^{\infty}\left[\frac{1}{\left(\alpha^{2}+\bar{R}^{2}\right)}\left(\frac{\beta_{1} \alpha\left(\bar{c}_{44} \alpha+\bar{R}^{2}\right)}{\left(\xi-\xi^{\prime \prime}\right)^{2}+\beta_{1}^{2} y^{2}}+\frac{\beta_{2} \bar{R}^{2}\left(\bar{c}_{44}-\alpha\right)}{\left(\xi-\xi^{\prime \prime}\right)^{2}+\beta_{2}^{2} y^{2}}\right)-\frac{e_{15}^{2}}{\varepsilon_{11}\left(\left(\xi-\xi^{\prime \prime}\right)^{2}+y^{2}\right)}\right] f\left(\xi^{\prime \prime}\right) \mathrm{d} \xi^{\prime \prime} \\
& -\frac{d y}{2 \pi} \int_{-\infty}^{\infty}\left[\frac{e_{15} e_{15}^{\prime}}{R}\left(\frac{\beta_{1}\left(\bar{c}_{44} \alpha+\bar{R}^{2}\right)}{\left(\alpha^{2}+\bar{R}^{2}\right)}\left(\frac{\beta_{2} \alpha\left(\bar{c}_{44}-\alpha\right)}{\left(\xi-\xi^{\prime \prime}\right)^{2}+\beta_{1}^{2} y^{2}}\right)-\frac{1}{\left(\xi-\xi^{\prime \prime}\right)^{2}+\beta_{2}^{2} y^{2}}\right)-\frac{1}{\varepsilon_{11}\left(\left(\xi-\xi^{\prime \prime}\right)^{2}+y^{2}\right)}\right] g\left(\xi^{\prime \prime}\right. \\
& -\frac{b_{4} y}{2 \pi} \int_{-\infty}^{\infty} \frac{e_{15}}{\left(\xi-\xi^{\prime \prime}\right)^{2}+y^{2}} j\left(\xi^{\prime \prime}\right) \mathrm{d} \xi^{\prime \prime} .
\end{aligned}
$$

Direct substitution into these of the representations (43)(45) for the densities yields apparently extremely unwieldy expressions in terms of double integrals for these stress components. But by first evaluating the integrals with respect to $\xi^{\prime \prime}$, using the results (A.1)-(A.8) outlined in Appendix, ultimately after extensive cumbersome manipulation and algebraic rearrangement they can be neatly written as

$$
\begin{aligned}
& \sigma_{y z}(\xi, y)=\mathcal{T}(\xi)+\bar{\Lambda}_{1} \mathcal{G}_{\beta_{1}}^{\mathcal{T}}\left(\theta_{\beta_{1}}\right)+\bar{\Lambda}_{2} \mathcal{G}_{\beta_{2}}^{\mathcal{T}}\left(\theta_{\beta_{2}}\right) \\
& +\bar{\Lambda}_{3}\left[\mathcal{G}_{\beta_{1}}^{\mathcal{H}}\left(\theta_{\beta_{1}}\right)-\mathcal{G}_{\beta_{2}}^{\mathcal{H}}\left(\theta_{\beta_{2}}\right)\right]+\bar{\Lambda}_{5} \mathcal{G}_{\beta_{1}}^{\mathcal{D}}\left(\theta_{\beta_{1}}\right) \\
& +\bar{\Lambda}_{6} \mathcal{G}_{\beta_{2}}^{\mathcal{D}}\left(\theta_{\beta_{2}}\right)-\frac{e_{15}}{\varepsilon_{11}} \mathcal{G}_{1}^{\mathcal{D}}\left(\theta_{1}\right), \\
& \sigma_{x z}(\xi, y)=-\frac{\bar{\Lambda}_{1}}{\beta_{1}} \mathcal{G}_{\beta_{1}}^{\mathcal{T}}\left(\theta_{\beta_{1}}-\frac{\pi}{2}\right)-\frac{\bar{\Lambda}_{2}}{\beta_{2}} \mathcal{G}_{\beta_{2}}^{\mathcal{T}}\left(\theta_{\beta_{2}}-\frac{\pi}{2}\right) \\
& -\bar{\Lambda}_{3}\left[\frac{1}{\beta_{1}} \mathcal{G}_{\beta_{1}}^{\mathcal{H}}\left(\theta_{\beta_{1}}-\frac{\pi}{2}\right)-\frac{1}{\beta_{2}} \mathcal{G}_{\beta_{2}}^{\mathcal{H}}\left(\theta_{\beta_{2}}-\frac{\pi}{2}\right)\right] \\
& \quad-\frac{\bar{\Lambda}_{5}}{\beta_{1}} \mathcal{G}_{\beta_{1}}^{\mathcal{D}}\left(\theta_{\beta_{1}}-\frac{\pi}{2}\right)-\frac{\bar{\Lambda}_{6}}{\beta_{2}} \mathcal{G}_{\beta_{2}}^{\mathcal{D}}\left(\theta_{\beta_{2}}-\frac{\pi}{2}\right) \\
& +\frac{e_{15}}{\varepsilon_{11}} \mathcal{G}_{1}^{\mathcal{D}}\left(\theta_{1}-\frac{\pi}{2}\right) .
\end{aligned}
$$

Analogously Eqs. (28)-(31) together with Eqs. (43)-(45) and (A.1)-(A.8) yield

$$
\begin{aligned}
& H_{z y}(\xi, y)=\mathcal{H}(\xi)+\bar{\Lambda}_{4}\left[\mathcal{G}_{\beta_{1}}^{\mathcal{T}}\left(\theta_{\beta_{1}}\right)-\mathcal{G}_{\beta_{2}}^{\mathcal{T}}\left(\theta_{\beta_{2}}\right)\right] \\
& +\bar{\Lambda}_{2} \mathcal{G}_{\beta_{1}}^{\mathcal{H}}\left(\theta_{\beta_{1}}\right)+\bar{\Lambda}_{1} \mathcal{G}_{\beta_{2}}^{\mathcal{H}}\left(\theta_{\beta_{2}}\right)+\bar{\Lambda}_{7} \mathcal{G}_{\beta_{1}}^{\mathcal{D}}\left(\theta_{\beta_{1}}\right) \\
& +\bar{\Lambda}_{8} \mathcal{G}_{\beta_{2}}^{\mathcal{D}}\left(\theta_{\beta_{2}}\right)-\frac{e_{15}^{\prime}}{\varepsilon_{11}} \mathcal{G}_{1}^{\mathcal{D}}\left(\theta_{1}\right),
\end{aligned}
$$

$$
\begin{aligned}
& H_{z x}(\xi, y)=-\bar{\Lambda}_{4}\left[\frac{1}{\beta_{1}} \mathcal{G}_{\beta_{1}}^{\mathcal{T}}\left(\theta_{\beta_{1}}-\frac{\pi}{2}\right)-\frac{1}{\beta_{2}} \mathcal{G}_{\beta_{2}}^{\mathcal{T}}\left(\theta_{\beta_{2}}-\frac{\pi}{2}\right)\right] \\
& \quad-\frac{\bar{\Lambda}_{2}}{\beta_{1}} \mathcal{G}_{\beta_{1}}^{\mathcal{H}}\left(\theta_{\beta_{1}}-\frac{\pi}{2}\right)-\frac{\bar{\Lambda}_{1}}{\beta_{2}} \mathcal{G}_{\beta_{2}}^{\mathcal{H}}\left(\theta_{\beta_{2}}-\frac{\pi}{2}\right) \\
& \quad-\frac{\bar{\Lambda}_{7}}{\beta_{1}} \mathcal{G}_{\beta_{1}}^{\mathcal{D}}\left(\theta_{\beta_{1}}-\frac{\pi}{2}\right)-\frac{\bar{\Lambda}_{8}}{\beta_{2}} \mathcal{G}_{\beta_{2}}^{\mathcal{D}}\left(\theta_{\beta_{2}}-\frac{\pi}{2}\right) \\
& \quad+\frac{e_{15}^{\prime}}{\varepsilon_{11}} \mathcal{G}_{1}^{\mathcal{D}}\left(\theta_{1}-\frac{\pi}{2}\right), \\
& D_{y}(\xi, y)=\mathcal{D}(\xi)+\mathcal{G}_{1}^{\mathcal{D}}\left(\theta_{1}\right), \\
& D_{x}(\xi, y)=\mathcal{G}_{1}^{\mathcal{D}}\left(\theta_{1}-\frac{\pi}{2}\right) .
\end{aligned}
$$

Here the functions $\mathcal{G}_{k}^{F}\left(\theta_{k}\right), \mathcal{R}_{k}(\xi, y)$ and $\theta_{k}(\xi, y)$ have been introduced for $F(\xi)=\mathcal{T}(\xi), \mathcal{H}(\xi)$ and $\mathcal{D}(\xi)$ and $k=\beta_{1}, \beta_{2}$ and 1 through the definitions $\mathcal{G}_{k}^{F}\left(\theta_{k}\right)=$

$$
-\frac{1}{\pi \mathcal{R}_{k}} \int_{-a_{1}}^{a_{1}} \frac{\mathcal{N}_{1}\left(\xi, \xi_{1}^{\prime}, \theta_{k}\right)}{\mathcal{N}_{2}\left(\xi, \xi_{1}^{\prime}\right)}\left(a_{1}^{2}-\xi_{1}^{\prime 2}\right)^{\frac{1}{2}} F\left(\xi_{1}^{\prime}\right) \mathrm{d} \xi_{1}^{\prime},
$$

$\mathcal{R}_{k} \mathrm{e}^{\mathrm{i} \theta_{k}}=\left[a_{1}^{2}-\sin ^{2}\left(\frac{\pi(\xi+\mathrm{i} k y)}{2 h}\right)\right]^{\frac{1}{2}}$,

where

$$
\begin{gathered}
\mathcal{N}_{1}\left(\xi, \xi_{1}^{\prime}, \theta_{k}\right)=\left[\sin \left(\frac{\pi \xi}{2 h}\right) \cosh \left(\frac{\pi k y}{2 h}\right)-\xi_{1}^{\prime}\right] \sin \left(\theta_{k}\right) \\
+\cos \left(\frac{\pi \xi}{2 h}\right) \sinh \left(\frac{\pi k y}{2 h}\right) \cos \left(\theta_{k}\right),
\end{gathered}
$$




$$
\begin{aligned}
& \mathcal{N}_{2}\left(\xi, \xi_{1}^{\prime}\right)=\left[\sin \left(\frac{\pi \xi}{2 h}\right) \cosh \left(\frac{\pi k y}{2 h}\right)-\xi_{1}^{\prime}\right]^{2} \\
& +\cos ^{2}\left(\frac{\pi \xi}{2 h}\right) \sinh ^{2}\left(\frac{\pi k y}{2 h}\right),
\end{aligned}
$$

and in Eq. (55) the square root function has branches which are stipulated to ensure that $\theta_{k}$ is zero for $|\xi|<a$, $y=0+$, and by analytic continuation elsewhere. The dimensionless constants $\bar{\Lambda}_{i}$ are defined for $i=1, \ldots, 8$ by

$$
\begin{aligned}
& \bar{\Lambda}_{1}=\frac{\left(\bar{c}_{44} \alpha+\bar{R}^{2}\right)\left(\alpha \bar{K}-\bar{R}^{2}\right)}{\left(\alpha^{2}+\bar{R}^{2}\right)\left(\bar{c}_{44} \bar{K}-\bar{R}^{2}\right)} \\
& \bar{\Lambda}_{2}=\frac{\bar{R}^{2}\left(\bar{c}_{44}-\alpha\right)(\alpha+\bar{K})}{\left(\alpha^{2}+\bar{R}^{2}\right)\left(\bar{c}_{44} \bar{K}-\bar{R}^{2}\right)}, \\
& \bar{\Lambda}_{3}=\frac{\bar{R}\left(\bar{c}_{44} \alpha+\bar{R}^{2}\right)\left(\bar{c}_{44}-\alpha\right)}{\left(\alpha^{2}+\bar{R}^{2}\right)\left(\bar{c}_{44} \bar{K}-\bar{R}^{2}\right)}, \\
& \bar{\Lambda}_{4}=\frac{\bar{R}(\alpha+\bar{K})\left(\alpha \bar{K}-\bar{R}^{2}\right)}{\left(\alpha^{2}+\bar{R}^{2}\right)\left(\bar{c}_{44} \bar{K}-\bar{R}^{2}\right)}, \\
& \bar{\Lambda}_{5}=\frac{\left(\bar{c}_{44} \alpha+\bar{R}^{2}\right)\left[e_{15}^{\prime} \bar{R}\left(\bar{c}_{44}-\alpha\right)+e_{15}\left(\alpha \bar{K}-\bar{R}^{2}\right)\right]}{\varepsilon_{11}\left(\alpha^{2}+\bar{R}^{2}\right)\left(\bar{c}_{44} \bar{K}-\bar{R}^{2}\right)}, \\
& \bar{\Lambda}_{6}=-\frac{\bar{R}\left(\bar{c}_{44}-\alpha\right)\left[e_{15}^{\prime}\left(\bar{c}_{44} \alpha+\bar{R}^{2}\right)-e_{15} \bar{R}(\alpha+\bar{K})\right]}{\varepsilon_{11}\left(\alpha^{2}+\bar{R}^{2}\right)\left(\bar{c}_{44} \bar{K}-\bar{R}^{2}\right)}, \\
& \bar{\Lambda}_{7}=\frac{\bar{R}(\alpha+\bar{K})\left[e_{15}^{\prime} \bar{R}\left(\bar{c}_{44}-\alpha\right)+e_{15}\left(\alpha \bar{K}-\bar{R}^{2}\right)\right]}{\varepsilon_{11}\left(\alpha^{2}+\bar{R}^{2}\right)\left(\bar{c}_{44} \bar{K}-\bar{R}^{2}\right)}, \\
& \bar{\Lambda}_{8}=\frac{\left(\alpha \bar{K}-\bar{R}^{2}\right)\left[e_{15}^{\prime}\left(\bar{c}_{44} \alpha+\bar{R}^{2}\right)-e_{15} \bar{R}(\alpha+\bar{K})\right]}{\varepsilon_{11}\left(\alpha^{2}+\bar{R}^{2}\right)\left(\bar{c}_{44} \bar{K}-\bar{R}^{2}\right)} .
\end{aligned}
$$

It is can be shown that

$\bar{\Lambda}_{1}+\bar{\Lambda}_{2}=1, \quad \Lambda_{5}+\bar{\Lambda}_{6}=\frac{e_{15}}{\varepsilon_{11}}, \quad \bar{\Lambda}_{7}+\bar{\Lambda}_{8}=\frac{e_{15}^{\prime}}{\varepsilon_{11}}$.

The phonon and phason stress components are observed from Eqs. (48)-(51) to depend upon all the loads applied, $\mathcal{T}(\xi), \mathcal{H}(\xi)$ and $\mathcal{D}(\xi)$, and the speed of the crack, as well as the moduli of the piezoelectric quasicrystal and the geometric parameters $a$ and $h$, with the boundary conditions (17) imposed, whereas clearly from Eqs. (52) and (53) the electric displacement components are decoupled and not dependent upon $\mathcal{T}(\xi)$ and $\mathcal{H}(\xi)$.

A demonstration of the distribution with angle close to a crack tip of these components, which is a feature of practical importance, is available by introducing polar coordinates $r$ and $\psi$, when $r \ll a$ such that

$$
\xi=a+r \cos \psi, \quad y=r \sin \psi
$$

It follows from Eq. (55) that approximately, as $r \rightarrow 0$,

$R_{k} \sim$

$\left[r \frac{\pi}{h} \sin \left(\frac{\pi a}{2 h}\right) \cos \left(\frac{\pi a}{2 h}\right)\left(\cos ^{2} \psi+k^{2} \sin ^{2} \psi\right)^{\frac{1}{2}}\right]^{\frac{1}{2}}$

$\theta_{k} \sim-\left(\pi-\Phi_{k}\right) / 2$ where

$$
\Phi_{k}=\tan ^{-1}(k \tan \psi),
$$

with the principal value of the inverse tangent, $\tan ^{-1}$ (...), chosen for $0 \leq \psi<\pi / 2$ and $\pi$ plus the principal value for $\pi / 2 \leq \psi \leq \pi$. Then, with

$$
\Delta_{k}=\left(\cos ^{2} \psi+k^{2} \sin ^{2} \psi\right)^{\frac{1}{4}},
$$

for $k=\beta_{1}, \beta_{2}$ and 1, it follows from Eqs. (48)-(57) that as $r \rightarrow 0$ :

$$
\begin{aligned}
\sigma_{y z}(r, \psi) & \sim \frac{K_{\mathcal{T}}^{R}}{\sqrt{r}}\left[\frac{\bar{\Lambda}_{1}}{\Delta_{\beta_{1}}} \cos \left(\frac{\Phi_{\beta_{1}}}{2}\right)+\frac{\bar{\Lambda}_{2}}{\Delta_{\beta_{2}}} \cos \left(\frac{\Phi_{\beta_{2}}}{2}\right)\right] \\
+ & \frac{K_{\mathcal{H}}^{R}}{\sqrt{r}} \bar{\Lambda}_{3}\left[\frac{1}{\Delta_{\beta_{1}}} \cos \left(\frac{\Phi_{\beta_{1}}}{2}\right)-\frac{1}{\Delta_{\beta_{2}}} \cos \left(\frac{\Phi_{\beta_{2}}}{2}\right)\right] \\
+ & \frac{K_{\mathcal{D}}^{R}}{\sqrt{r}}\left[\frac{\bar{\Lambda}_{5}}{\Delta_{\beta_{1}}} \cos \left(\frac{\Phi_{\beta_{1}}}{2}\right)+\frac{\bar{\Lambda}_{6}}{\Delta_{\beta_{2}}} \cos \left(\frac{\Phi_{\beta_{2}}}{2}\right)\right. \\
& \left.-\frac{e_{15}}{\varepsilon_{11}} \cos \left(\frac{\psi}{2}\right)\right],
\end{aligned}
$$

$$
\begin{aligned}
H_{z y}(r, \psi) & \sim \frac{K_{\mathcal{T}}^{R}}{\sqrt{r}} \bar{\Lambda}_{4}\left[\frac{1}{\Delta_{\beta_{1}}} \cos \left(\frac{\Phi_{\beta_{1}}}{2}\right)-\frac{1}{\Delta_{\beta_{2}}} \cos \left(\frac{\Phi_{\beta_{2}}}{2}\right)\right] \\
+ & \frac{K_{\mathcal{H}}^{R}}{\sqrt{r}}\left[\frac{\bar{\Lambda}_{2}}{\Delta_{\beta_{1}}} \cos \left(\frac{\Phi_{\beta_{1}}}{2}\right)+\frac{\bar{\Lambda}_{1}}{\Delta_{\beta_{2}}} \cos \left(\frac{\Phi_{\beta_{2}}}{2}\right)\right] \\
+ & \frac{K_{\mathcal{D}}^{R}}{\sqrt{r}}\left[\frac{\bar{\Lambda}_{7}}{\Delta_{\beta_{1}}} \cos \left(\frac{\Phi_{\beta_{1}}}{2}\right)+\frac{\bar{\Lambda}_{8}}{\Delta_{\beta_{2}}} \cos \left(\frac{\Phi_{\beta_{2}}}{2}\right)\right. \\
- & \left.\frac{e_{15}^{\prime}}{\varepsilon_{11}} \cos \left(\frac{\psi}{2}\right)\right], \\
D_{y}(r, \psi) & \sim \frac{K_{\mathcal{D}}^{R}}{\sqrt{r}} \cos \left(\frac{\psi}{2}\right),
\end{aligned}
$$

$$
\sigma_{x z}(r, \psi) \sim-\frac{K_{T}^{R}}{\sqrt{r}}\left[\frac{\bar{\Lambda}_{1}}{\beta_{1} \Delta_{\beta_{1}}} \sin \left(\frac{\Phi_{\beta_{1}}}{2}\right)+\frac{\bar{\Lambda}_{2}}{\beta_{2} \Delta_{\beta_{2}}} \sin \left(\frac{\Phi_{\beta_{2}}}{2}\right)\right]
$$$$
-\frac{K_{\mathcal{H}}^{R}}{\sqrt{r}} \bar{\Lambda}_{3}\left[\frac{1}{\beta_{1} \Delta_{\beta_{1}}} \sin \left(\frac{\Phi_{\beta_{1}}}{2}\right)-\frac{1}{\beta_{2} \Delta_{\beta_{2}}} \sin \left(\frac{\Phi_{\beta_{2}}}{2}\right)\right]
$$$$
-\frac{K_{\mathcal{D}}^{R}}{\sqrt{r}}\left[\frac{\bar{\Lambda}_{5}}{\beta_{1} \Delta_{\beta_{1}}} \sin \left(\frac{\Phi_{\beta_{1}}}{2}\right)+\frac{\bar{\Lambda}_{6}}{\beta_{2} \Delta_{\beta_{2}}} \sin \left(\frac{\Phi_{\beta_{2}}}{2}\right)\right.
$$$$
\left.-\frac{e_{15}}{\varepsilon_{11}} \sin \left(\frac{\psi}{2}\right)\right] \text {, }
$$

$H_{z x}(r, \psi) \sim$

$$
\begin{aligned}
& -\frac{K_{\mathcal{T}}^{R}}{\sqrt{r}} \bar{\Lambda}_{4}\left[\frac{1}{\beta_{1} \Delta_{\beta_{1}}} \sin \left(\frac{\Phi_{\beta_{1}}}{2}\right)-\frac{1}{\beta_{2} \Delta_{\beta_{2}}} \sin \left(\frac{\Phi_{\beta_{2}}}{2}\right)\right] \\
& -\frac{K_{\mathcal{H}}^{R}}{\sqrt{r}}\left[\frac{\bar{\Lambda}_{2}}{\beta_{1} \Delta_{\beta_{1}}} \sin \left(\frac{\Phi_{\beta_{1}}}{2}\right)+\frac{\bar{\Lambda}_{1}}{\beta_{2} \Delta_{\beta_{2}}} \sin \left(\frac{\Phi_{\beta_{2}}}{2}\right)\right] \\
& -\frac{K_{\mathcal{D}}^{R}}{\sqrt{r}}\left[\frac{\bar{\Lambda}_{7}}{\beta_{1} \Delta_{\beta_{1}}} \sin \left(\frac{\Phi_{\beta_{1}}}{2}\right)+\frac{\bar{\Lambda}_{8}}{\beta_{2} \Delta_{\beta_{2}}} \sin \left(\frac{\Phi_{\beta_{2}}}{2}\right)\right. \\
& \left.-\frac{e_{15}^{\prime}}{\varepsilon_{11}} \sin \left(\frac{\psi}{2}\right)\right],
\end{aligned}
$$




$$
D_{x}(r, \psi) \sim \frac{K_{\mathcal{D}}^{R}}{\sqrt{r}} \sin \left(\frac{\psi}{2}\right) .
$$

Here $K_{\mathcal{T}}^{R}, K_{\mathcal{H}}^{R}$ and $K_{\mathcal{D}}^{R}$ are the appropriate phonon and phason stress and electric displacement intensity factors at the tip of a crack within a row of cracks, given for $F(\xi)=\mathcal{T}(\xi), \mathcal{H}(\xi)$ or $\mathcal{D}(\xi)$ by

$$
\begin{aligned}
K_{F}^{R} & =\frac{1}{2}\left[\frac{1}{\pi h \sin \left(\frac{\pi a}{2 h}\right) \cos \left(\frac{\pi a}{2 h}\right)}\right]^{\frac{1}{2}} \\
& \times \int_{-a}^{a}\left[\frac{\sin \left(\frac{\pi a}{2 h}\right)+\sin \left(\frac{\pi \xi^{\prime}}{2 h}\right)}{\sin \left(\frac{\pi a}{2 h}\right)-\sin \left(\frac{\pi \xi^{\prime}}{2 h}\right)}\right]^{\frac{1}{2}} \cos \left(\frac{\pi \xi^{\prime}}{2 h}\right) F\left(\xi^{\prime}\right) \mathrm{d} \xi^{\prime} .
\end{aligned}
$$

It is noteworthy that, as would be expected, in the limit as $h \rightarrow \infty, K_{F}^{R} \rightarrow K_{F}$, where

$$
K_{F}=\frac{1}{\pi \sqrt{2 a}} \int_{-a}^{a}\left(\frac{a+\xi^{\prime}}{a-\xi^{\prime}}\right)^{\frac{1}{2}} F\left(\xi^{\prime}\right) \mathrm{d} \xi^{\prime},
$$

are the corresponding intensity factors for an isolated non-uniformly loaded, single Griffith crack; in agreement with those derived by Tupholme [23].

Further, when the cracks are subject to uniform loads, so that $F(\xi)=F=$ constant, the integrals in Eqs. (76) and $(77)$ can be evaluated explicitly and $K_{F}^{R}$ and $K_{F}$, respectively, then reduce to

$$
\begin{aligned}
K_{F}^{R} & =\left[\frac{h}{\pi} \tan \left(\frac{\pi a}{2 h}\right)\right]^{\frac{1}{2}} F, \\
K_{F} & =\left(\frac{a}{2}\right)^{\frac{1}{2}} F .
\end{aligned}
$$

The expression (78) agrees when $F=\mathcal{T}=$ constant with the classical stress intensity factor of a row of cracks subjected to a uniform load $\mathcal{T}$ in purely elastic isotropic media, as first derived by Sneddon and Lowengrub [2].
Hence when uniform loads $\mathcal{T}(\xi)=T=$ constant, $\mathcal{H}(\xi)=H=$ constant and $\mathcal{D}(\xi)=D=$ constant are imposed

$$
K_{F}^{R}=\left[\frac{\tan (\pi a / 2 h)}{\pi a / 2 h}\right]^{\frac{1}{2}} K_{F},
$$

for $F=T, H$ and $D$, and thus then the intensity factors at a crack tip in the row are each of larger magnitudes than the corresponding values near an analogous single uniformly-loaded crack and are larger the higher the value of $a / h$. The variation of the normalized field intensity factors, $K_{F}^{R} / K_{F}$, of a uniformly-loaded row with the relative spacing $a / h$ is depicted in Fig. 2.

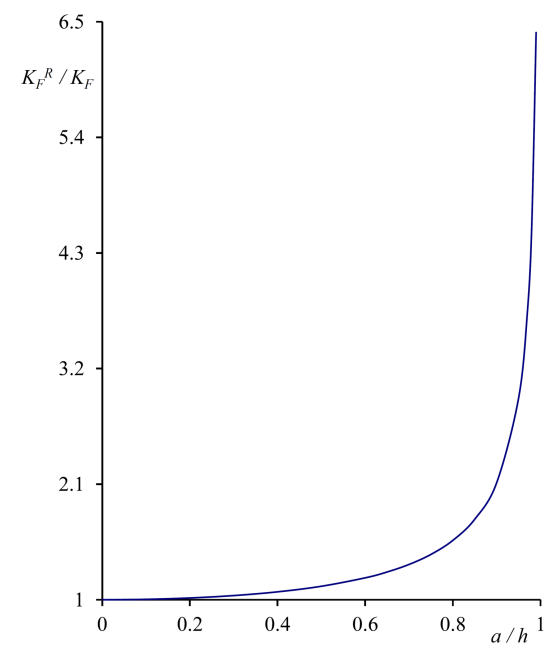

Fig. 2. Variation of the normalized field intensity factors $K_{F}^{R} / K_{F}$ with the relative spacing of a row of uniformly-loaded cracks.

Moreover, by combining Eqs. (70) and (73), (71) and (74), and (72) and (75), respectively, the polar components $\sigma_{\psi z}$ and $H_{z \psi}$ of the phonon and phason stress and $D_{\psi}$ of the electric displacement near the crack tip become

$$
\begin{aligned}
& \sigma_{\psi z}(r, \psi) \sim \frac{\bar{\Lambda}_{1} K_{\mathcal{T}}^{R}+\bar{\Lambda}_{3} K_{\mathcal{H}}^{R}+\bar{\Lambda}_{5} K_{\mathcal{D}}^{R}}{\sqrt{r} \Delta_{\beta_{1}}}\left[\frac{1}{\beta_{1}} \sin \left(\frac{\Phi_{\beta_{1}}}{2}\right) \sin \psi+\cos \left(\frac{\Phi_{\beta_{1}}}{2}\right) \cos \psi\right] \\
&+\frac{\bar{\Lambda}_{2} K_{\mathcal{T}}^{R}-\bar{\Lambda}_{3} K_{\mathcal{H}}^{R}+\bar{\Lambda}_{6} K_{\mathcal{D}}^{R}}{\sqrt{r} \Delta_{\beta_{2}}}\left[\frac{1}{\beta_{2}} \sin \left(\frac{\Phi_{\beta_{2}}}{2}\right) \sin \psi+\cos \left(\frac{\Phi_{\beta_{2}}}{2}\right) \cos \psi\right]-\frac{e_{15} K_{\mathcal{D}}^{R}}{\varepsilon_{11} \sqrt{r}} \cos \left(\frac{\psi}{2}\right), \\
& H_{z \psi}(r, \psi) \sim \frac{\bar{\Lambda}_{4} K_{\mathcal{T}}^{R}+\bar{\Lambda}_{2} K_{\mathcal{H}}^{R}+\bar{\Lambda}_{7} K_{\mathcal{D}}^{R}}{\sqrt{r} \Delta_{\beta_{1}}}\left[\frac{1}{\beta_{1}} \sin \left(\frac{\Phi_{\beta_{1}}}{2}\right) \sin \psi+\cos \left(\frac{\Phi_{\beta_{1}}}{2}\right) \cos \psi\right] \\
&-\frac{\bar{\Lambda}_{4} K_{\mathcal{T}}^{R}-\bar{\Lambda}_{1} K_{\mathcal{H}}^{R}-\bar{\Lambda}_{8} K_{\mathcal{D}}^{R}}{\sqrt{r} \Delta_{\beta_{2}}}\left[\frac{1}{\beta_{2}} \sin \left(\frac{\Phi_{\beta_{2}}}{2}\right) \sin \psi+\cos \left(\frac{\Phi_{\beta_{2}}}{2}\right) \cos \psi\right]-\frac{e_{15}^{\prime} K_{\mathcal{D}}^{R}}{\varepsilon_{11} \sqrt{r}} \cos \left(\frac{\psi}{2}\right),
\end{aligned}
$$

$$
D_{\psi}(r, \psi) \sim \frac{K_{\mathcal{D}}^{R}}{\sqrt{r}} \cos \left(\frac{\psi}{2}\right) .
$$

These demonstrate that the components are all governed by a classical $1 / \sqrt{r}$ crack-tip behaviour and that their dependence upon the non-uniform loads is only through the intensity factors given by Eq. (76).

Further, it was observed above that all the density functions given by Eqs. (43)-(45) are dependent upon the loads applied together with the speed and geometrical and material constants of the situation. But in contrast the intensity factors, given by Eq. (76), depend only upon the applied loads $\mathcal{T}(\xi), \mathcal{H}(\xi)$ and $\mathcal{D}(\xi)$, respectively, and $a$ and $h$.

It should be mentioned that, from Eqs. (43)-(45), the above analysis is not valid when $\bar{c}_{44} \bar{K}-\bar{R}^{2}=0$ or $\beta_{1}=0$ or $\beta_{2}=0$. The value of $\beta_{i}$ is seen from the definition (23) to be zero when the speed of the crack, $v$, equals the wave speed $s_{i}$, for $i=1,2$. 
The reported values of the material constants data of one-dimensional hexagonal piezoelectric quasicrystals are still not completely reliable. But typically Li et al. [15] report $c_{44}=5.0 \times 10^{10} \mathrm{~N} \mathrm{~m}^{-2}, R=1.2 \times 10^{9} \mathrm{~N} \mathrm{~m}^{-2}$, $K=3.0 \times 10^{8} \mathrm{~N} \mathrm{~m}^{-2}, e_{15}=-0.138 \mathrm{C} \mathrm{m}^{-2}$, $e_{15}^{\prime}=-0.160 \mathrm{C} \mathrm{m}^{-2}, \varepsilon_{11}=82.6 \times 10^{-12} \mathrm{C}^{2} \mathrm{~N}^{-1} \mathrm{~m}^{-2}$, and typically $\rho=5.1 \times 10^{3} \mathrm{~kg} \mathrm{~m}^{-3}$. Then the wave speeds are $s_{1} \approx 3139 \mathrm{~ms}^{-1}$ and $s_{2} \approx 333 \mathrm{~ms}^{-1}$. Moreover, clearly $c_{44} \bar{K}-\bar{R}^{2}$ is non-zero, since the value of $\bar{R}^{2}$ is much smaller than $\bar{c}_{44} \bar{K}$.

Illustrative curves are shown in Fig. 3 for the variation with the angle $\psi$ around the crack tip of the scaled phonon stress component, $\sqrt{r} \sigma_{\psi z} / K_{\mathcal{T}}^{R}$, as calculated from Eq. (81) with the material moduli data given above for $0 \leq v / s_{2}<1$ for a representative electrically impermeable crack with $\mathcal{H}(\xi)=\mathcal{D}(\xi)=0$. The graphs presented demonstrate that as $\psi$ increases from zero at a particular speed there is a decrease in the magnitude of this stress component; with the magnitude of this decrease being smaller than that around the tip of a stationary crack. The variation with the angle $\psi$ of the corresponding scaled phason stress component, $-\sqrt{r} H_{z \psi} / K_{\mathcal{T}}^{R}$, as calculated from Eq. (82), is illustrated in Fig. 4 for a range of values of $v / s_{2}$. It is seen that this phason component has a magnitude that is significantly smaller than that of the phonon component. Clearly, from Eq. (82), in this situation the electric displacement component is identically zero because of the previouslynoted decoupling.

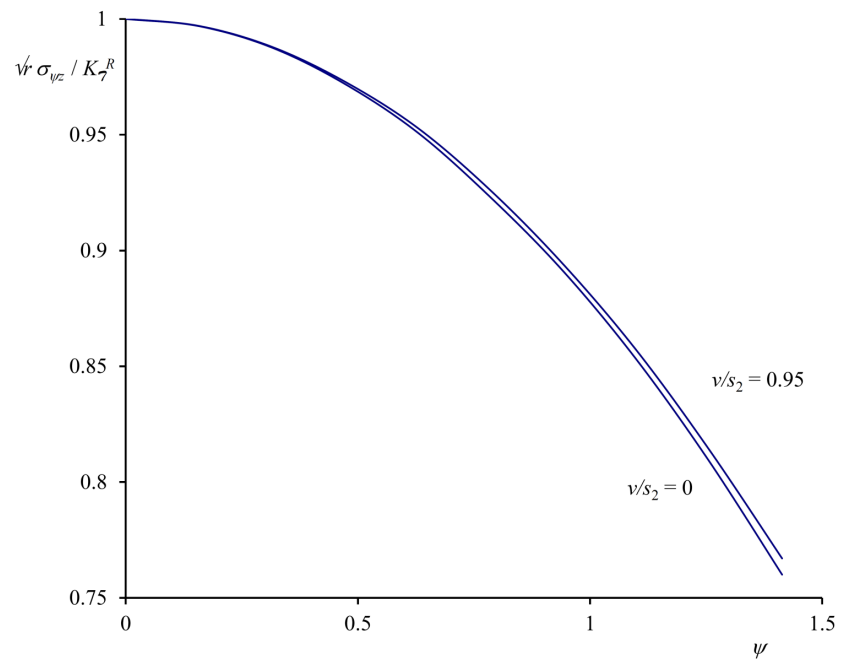

Fig. 3. Distribution of the scaled phonon stress component $\sqrt{r} \sigma_{\psi z} / K_{\mathcal{T}}^{R}$ around a crack tip for the scaled speeds $v / s_{2}=0$ and $v / s_{2}=0.95$ in a piezoelectric quasicrystal.

An indication of the difference in the value of the scaled phonon stress component $\sqrt{r} \sigma_{\psi z} / K_{\mathcal{T}}^{R}$ at a speed $v$ from its value when $v=0$ is shown in Fig. 5 . The variation around the crack tip of $\sqrt{r} \tau_{\psi z} / K_{\mathcal{T}}^{R}$ is displayed for a range of values of $v / s_{2}$, where $\tau_{\psi z}=10^{3}\left(\sigma_{\psi z}-\left.\sigma_{\psi z}\right|_{v=0}\right)$.

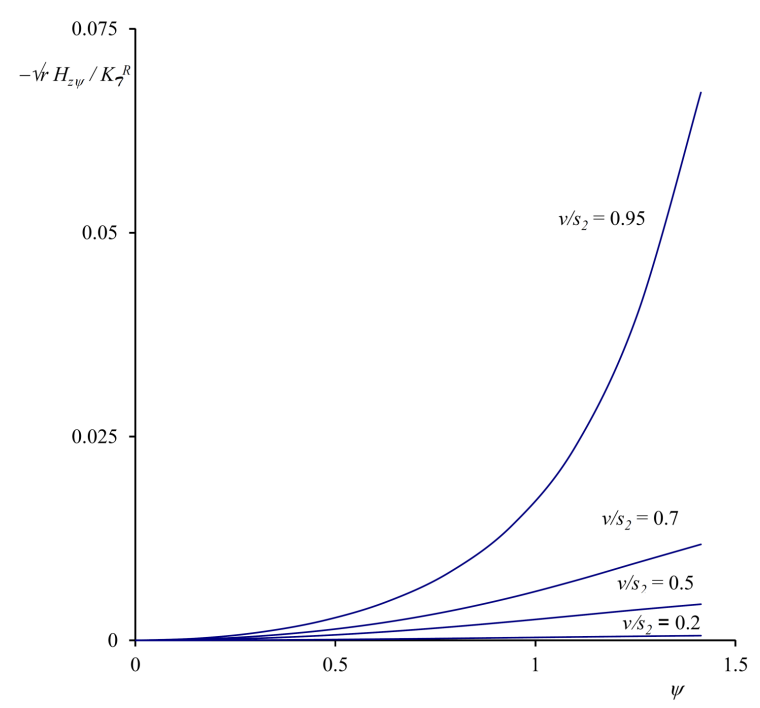

Fig. 4. Distribution of the scaled phason stress component $-\sqrt{r} H_{z \psi} / K_{\mathcal{T}}^{R}$ around a crack tip for a range of values of the scaled speed $v / s_{2}$ in a piezoelectric quasicrystal.

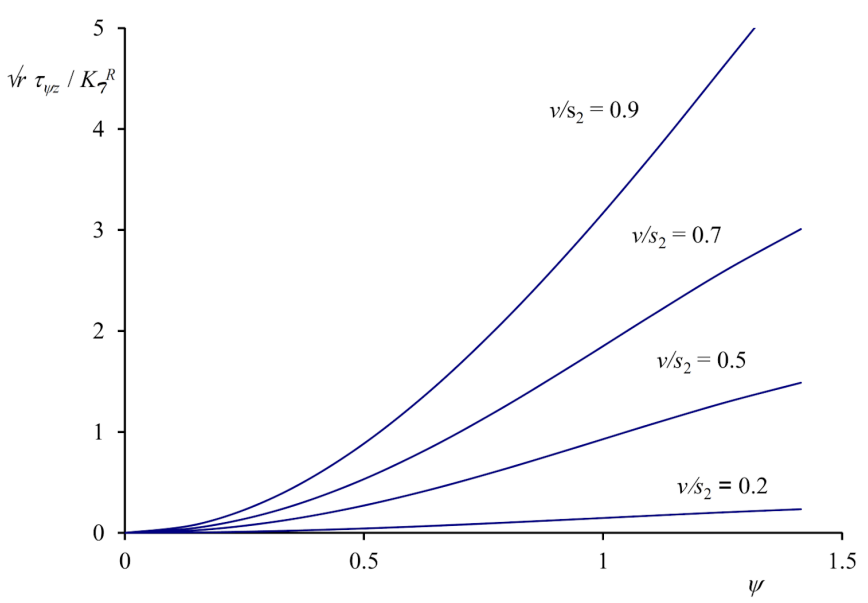

Fig. 5. Distribution of the scaled phonon stress component $\sqrt{r} \tau_{\psi z} / K_{\mathcal{T}}^{R}$ around a crack tip for a range of values of the scaled speed $v / s_{2}$ in a piezoelectric quasicrystal.

\section{Stationary row of non-uniformly loaded shear cracks in piezoelectric quasicrystals}

There has been no presentation of the analogous analysis for a stationary row of cracks in a piezoelectric quasicrystal which can be deduced by putting $v=0$ throughout the above. The resulting components of the fields are much simplified.

When $v=0$, from Eq. (23), $\beta_{1}=\beta_{2}=1$ and recollecting that, from Eqs. (64), $\bar{\Lambda}_{1}+\bar{\Lambda}_{2}=1, \bar{\Lambda}_{5}+\bar{\Lambda}_{6}=e_{15} / \varepsilon_{11}$ and $\bar{\Lambda}_{7}+\bar{\Lambda}_{8}=e_{15}^{\prime} / \varepsilon_{11}$, Eqs. (48)-(53) reduce to 
$\sigma_{y z}(x, y)=\mathcal{T}(x)+\mathcal{G}_{1}^{\mathcal{T}}\left(\theta_{1}\right), H_{z y}(x, y)=\mathcal{H}(x)+\mathcal{G}_{1}^{\mathcal{H}}\left(\theta_{1}\right)$,

$D_{y}(x, y)=\mathcal{D}(x)+\mathcal{G}_{1}^{\mathcal{D}}\left(\theta_{1}\right)$,

$\sigma_{x z}(x, y)=-\mathcal{G}_{1}^{\mathcal{T}}\left(\theta_{1}-\frac{\pi}{2}\right), H_{z x}(x, y)=-\mathcal{G}_{1}^{\mathcal{H}}\left(\theta_{1}-\frac{\pi}{2}\right)$

$D_{x}(x, y)=-\mathcal{G}_{1}^{\mathcal{D}}\left(\theta_{1}-\frac{\pi}{2}\right)$.

Moreover, as $r \rightarrow 0$, Eqs. (70)-(75) and (81)-(83) yield

$\sigma_{y z}(r, \psi) \sim \frac{K_{\mathcal{T}}^{R}}{\sqrt{r}} \cos \left(\frac{\psi}{2}\right), \quad H_{z y}(r, \psi) \sim \frac{K_{\mathcal{H}}^{R}}{\sqrt{r}} \cos \left(\frac{\psi}{2}\right)$,

$D_{y}(r, \psi) \sim \frac{K_{\mathcal{D}}^{R}}{\sqrt{r}} \cos \left(\frac{\psi}{2}\right)$,

$\sigma_{x z}(r, \psi) \sim-\frac{K_{\mathcal{T}}^{R}}{\sqrt{r}} \sin \left(\frac{\psi}{2}\right), H_{z x}(r, \psi) \sim-\frac{K_{\mathcal{H}}^{R}}{\sqrt{r}} \sin \left(\frac{\psi}{2}\right)$,

$D_{x}(r, \psi) \sim-\frac{K_{\mathcal{D}}^{R}}{\sqrt{r}} \sin \left(\frac{\psi}{2}\right)$,

and

$\sigma_{\psi z}(r, \psi) \sim \frac{K_{\mathcal{T}}^{R}}{\sqrt{r}} \cos \left(\frac{\psi}{2}\right), \quad H_{z \psi}(r, \psi) \sim \frac{K_{\mathcal{H}}^{R}}{\sqrt{r}} \cos \left(\frac{\psi}{2}\right)$

$D_{\psi}(r, \psi) \sim \frac{K_{\mathcal{D}}^{R}}{\sqrt{r}} \cos \left(\frac{\psi}{2}\right)$.

It is worthy of note that in Eqs. (81) and (82) the components $\sigma_{\psi z}(r, \psi)$ and $H_{z \psi}(r, \psi)$ around a moving crack both depend upon $\mathcal{T}(\xi), \mathcal{H}(\xi)$ and $\mathcal{D}(\xi)$, whereas in contrast for a stationary crack it can be seen from Eqs. (88) that $\sigma_{\psi z}(r, \psi)$ is dependent upon only $\mathcal{T}(x)$ and $H_{z \psi}(r, \psi)$ upon only $\mathcal{H}(x)$.

\section{Non-uniformly loaded row of moving shear cracks in hexagonal piezoelectric crystals}

No expressions have been given before for the components of the fields, or their variation with angle, around a row of non-uniformly loaded moving cracks in hexagonal piezoelectric crystals; nor even when the load is uniform and/or the cracks are stationary.

The analyses of Sects. 4 and 5 are simplified in the absence of a phason field to those for hexagonal piezoelectric crystals by putting $R=K=e_{15}^{\prime}=0$ throughout. Then $\bar{\Lambda}_{1}=1, \bar{\Lambda}_{5}=e_{15} / \varepsilon_{11}$ and $\bar{\Lambda}_{i}=0$ for $i=2,3,4,6,7$ and 8. In particular, Eq. (81) and (83) yield

$$
\begin{aligned}
& \sigma_{\psi z}(r, \psi) \sim \frac{K_{\mathcal{T}}^{R}}{\sqrt{r} \Delta_{\kappa}}\left[\frac{1}{\kappa} \sin \left(\frac{\Phi_{\kappa}}{2}\right) \sin \psi+\cos \left(\frac{\Phi_{\kappa}}{2}\right) \cos \psi\right] \\
&+\frac{K_{\mathcal{D}}^{R}}{\sqrt{r}} \frac{e_{15}}{\varepsilon_{11}}\left\{\frac{1}{\Delta_{\kappa}}\left[\frac{1}{\kappa} \sin \left(\frac{\Phi_{\kappa}}{2}\right) \sin \psi+\cos \left(\frac{\Phi_{\kappa}}{2}\right) \cos \psi\right]\right. \\
&\left.-\cos \left(\frac{\psi}{2}\right)\right\}, \\
& D_{\psi}(r, \psi) \sim \frac{K_{\mathcal{D}}^{R}}{\sqrt{r}} \cos \left(\frac{\psi}{2}\right),
\end{aligned}
$$

where, with $\mu_{e}$ and $v_{e}$ being the piezoelectrically stiffened elastic constant and bulk shear wave speed, respectively,

$$
\kappa=\sqrt{1-\frac{v^{2}}{v_{e}^{2}}}, \quad v_{e}=\sqrt{\frac{\mu_{e}}{\rho}}, \quad \mu_{e}=c_{44}+\frac{e_{15}^{2}}{\varepsilon_{11}}
$$

and $\Phi_{\kappa}$ and $\Delta_{\kappa}$ are given by Eqs. (68) and (69) with $k=\kappa$.

The results for a stationary row of cracks are given by Eqs. (89) and (90) with $\kappa=1$.

\section{Non-uniformly loaded row of moving shear cracks in isotropic elastic solids}

Finally, by removing the piezoelectric effects from the expressions in Sect. 6 by putting $e_{15}=0$, the results are reduced to those for a row of non-uniformly loaded moving cracks in a purely isotropic elastic. It is seen that, with $c_{44}=\mu$, the second Lamé constant,

$\sigma_{\psi z}(r, \psi) \sim$

$$
\frac{K_{\mathcal{T}}^{R}}{\sqrt{r} \Delta_{\beta}}\left[\frac{1}{\beta} \sin \left(\frac{\Phi_{\beta}}{2}\right) \sin \psi+\cos \left(\frac{\Phi_{\beta}}{2}\right) \cos \psi\right],
$$

where $\beta$ and the shear wave speed, $v_{s}$, are defined by:

$$
\beta=\sqrt{1-\frac{v^{2}}{v_{s}^{2}}}, \quad v_{s}=\sqrt{\frac{\mu}{\rho}},
$$

and $\Phi_{\beta}$ and $\Delta_{\beta}$ are given by Eqs. (68) and (69) with $k=\beta$.

The corresponding expression for a row of stationary crack follows, by putting $\beta=1$, as

$$
\sigma_{\psi z}(r, \psi) \sim \frac{K_{\mathcal{T}}^{R}}{\sqrt{r}} \cos \left(\frac{\psi}{2}\right) .
$$

The variation with the angle $\psi$ around a crack tip of the scaled stress component $\sqrt{r} \sigma_{\psi z} / K_{\mathcal{T}}^{R}$, given by Eq. (92), for a range of values of $v / v_{s}$ is depicted in Fig. 6. The graphs demonstrate that $\sigma_{\psi z}$ develops an off-axis max-

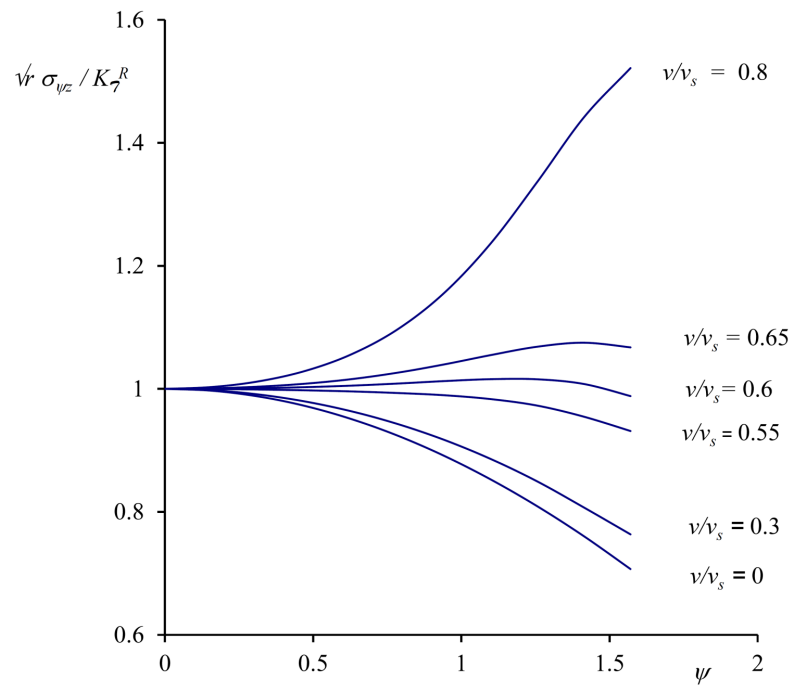

Fig. 6. Distribution of the scaled stress component $\sqrt{r} \sigma_{\psi z} / K_{\mathcal{T}}^{R}$ around a crack tip for a range of values of the scaled speed $v / v_{s}$ in an isotropic elastic material. 
imum (i.e. in a non-forward direction) for sufficiently high values of the speed $v$; which first occurs when $v / v_{s} \approx 0.6$. This indicates that these cracks have a tendency to branch when moving in a tearing mode.

\section{Conclusions}

The deformed fields around a non-uniformly loaded row of collinear mode III shear cracks in one-dimensional piezoelectric quasicrystals are investigated using an analysis which is fundamentally based upon an extension of the continuous dislocation arrays method.

The singular integral equations that must be satisfied by the appropriate densities of the array of piezoelectric quasicrystal screw dislocations are solved exactly. Analytic explicit representations then follow for the phonon, phason and electric field components. The intensity factors and the variations of the components with the angle near a crack tip are determined.

For representative material moduli and various crack speeds, the angular variations of the phonon stress component are illustrated graphically.

The corresponding results, which previously have not been available, for solid media that are special cases of these very general modern materials and constantlyloaded cracks are discussed.

\section{Appendix}

The representations (46) and (47) for the phonon stress components $\sigma_{y z}$ and $\sigma_{x z}$, for example, respectively involve integrals of the forms

$$
\begin{aligned}
& I_{k}(\xi, y)=\int_{-\infty}^{\infty} \frac{\left(\xi-\xi^{\prime \prime}\right)}{\left(\xi-\xi^{\prime \prime}\right)^{2}+k^{2} y^{2}} r\left(\xi^{\prime \prime}\right) \mathrm{d} \xi^{\prime \prime} \\
& J_{k}(\xi, y)=-\int_{-\infty}^{\infty} \frac{y}{\left(\xi-\xi^{\prime \prime}\right)^{2}+k^{2} y^{2}} r\left(\xi^{\prime \prime}\right) \mathrm{d} \xi^{\prime \prime}
\end{aligned}
$$

for $k=\beta_{1}, \beta_{2}$ and 1 and $r(\xi)=f(\xi), g(\xi)$ and $j(\xi)$.

It is straightforward and neat to evaluate these simultaneously by combining them as the complex function

$$
\begin{aligned}
K_{k}(\xi, y)=I_{k}(\xi, y)+\mathrm{i} k J_{k}(\xi, y)= \\
=\int_{-\infty}^{\infty} \frac{1}{\xi-\xi^{\prime \prime}+\mathrm{i} k y} r\left(\xi^{\prime \prime}\right) \mathrm{d} \xi^{\prime \prime} \\
=\int_{-a_{1}}^{-a_{1}} \frac{1}{\sin (\pi(\xi+\mathrm{i} k y) / 2 h)-\xi_{1}^{\prime \prime}} r_{1}\left(\xi_{1}^{\prime \prime}\right) \mathrm{d} \xi_{1}^{\prime \prime}
\end{aligned}
$$

where the same type of manipulation and corresponding notation as that utilized in replacing the infinite range integral in Eq. (36) by the finite range integral in Eq. (42) has been adopted to rewrite Eq. (A.3) as Eq. (A.4).

Substituting for $r_{1}\left(\xi_{1}^{\prime \prime}\right)$ from Eqs. (43), (44) or (45) shows that $K_{k}(\xi, y)$ consists of a sum of terms involving appropriate constant multiples of double integrals of the form

$$
\begin{gathered}
L_{k}(\xi, y)=\int_{-a_{1}}^{a_{1}}\left(a_{1}^{2}-\xi_{1}^{\prime 2}\right)^{\frac{1}{2}}\left\{\int_{-a_{1}}^{a_{1}} \frac{1}{\left(a_{1}^{2}-\xi_{1}^{\prime \prime 2}\right)^{\frac{1}{2}}\left(\xi_{1}^{\prime}-\xi_{1}^{\prime \prime}\right)}\right. \\
\left.\times \frac{1}{\sin \left(\frac{\pi(\xi+\mathrm{i} k y)}{2 h}\right)-\xi_{1}^{\prime \prime}} \mathrm{d} \xi_{1}^{\prime \prime}\right\} F\left(\xi_{1}^{\prime}\right) \mathrm{d} \xi_{1}^{\prime}
\end{gathered}
$$

with $F(\xi)=\mathcal{T}(\xi), \mathcal{H}(\xi)$ or $\mathcal{D}(\xi)$. The inner integral involved here can be conveniently evaluated by the methods of complex variable contour integration to yield

$L_{k}(\xi, y)=$

$$
-\frac{\mathrm{i} \pi \mathrm{e}^{-\mathrm{i} \theta_{k}}}{\mathcal{R}_{k}} \int_{-a_{1}}^{a_{1}} \frac{\left(a_{1}^{2}-\xi_{1}^{\prime 2}\right)^{\frac{1}{2}}}{\sin \left(\frac{\pi(\xi+\mathrm{i} k y)}{2 h}\right)-\xi_{1}^{\prime}} F\left(\xi_{1}^{\prime}\right) \mathrm{d} \xi_{1}^{\prime},
$$

where

$$
\mathcal{R}_{k} \mathrm{e}^{\mathrm{i} \theta_{k}}=\left[a_{1}^{2}-\sin ^{2}\left(\frac{\pi(\xi+\mathrm{i} k y)}{2 h}\right)\right]^{\frac{1}{2}}
$$

with its branches chosen as in Eq. (55). Finally, the real and imaginary parts of the representation (A.5) can be readily derived in terms of the function $\mathcal{G}_{k}^{F}\left(\theta_{k}\right)$ that is defined for $F(\xi)=\mathcal{T}(\xi), \mathcal{H}(\xi)$ and $\mathcal{D}(\xi)$ by

$\mathcal{G}_{k}^{F}\left(\theta_{k}\right)=$

$$
-\frac{1}{\pi \mathcal{R}_{k}} \int_{-a_{1}}^{a_{1}} \frac{\mathcal{N}_{1}\left(\xi, \xi_{1}^{\prime}, \theta_{k}\right)}{\mathcal{N}_{2}\left(\xi, \xi_{1}^{\prime}\right)}\left(a_{1}^{2}-\xi_{1}^{2}\right)^{\frac{1}{2}} F\left(\xi_{1}^{\prime}\right) \mathrm{d} \xi_{1}^{\prime},
$$

where

$$
\begin{aligned}
& \mathcal{N}_{1}\left(\xi, \xi_{1}^{\prime}, \theta_{k}\right)=\left[\sin \left(\frac{\pi \xi}{2 h}\right) \cosh \left(\frac{\pi k y}{2 h}\right)-\xi_{1}^{\prime}\right] \sin \left(\theta_{k}\right) \\
& +\cos \left(\frac{\pi \xi}{2 h}\right) \sinh \left(\frac{\pi k y}{2 h}\right) \cos \left(\theta_{k}\right), \\
& \mathcal{N}_{2}\left(\xi, \xi_{1}^{\prime}\right)=\left[\sin \left(\frac{\pi \xi}{2 h}\right) \cosh \left(\frac{\pi k y}{2 h}\right)-\xi_{1}^{\prime}\right]^{2} \\
& +\cos ^{2}\left(\frac{\pi \xi}{2 h}\right) \sinh ^{2}\left(\frac{\pi k y}{2 h}\right) .
\end{aligned}
$$

Alternatively, the integrals $I_{k}(\xi, y)$ and $J_{k}(\xi, y)$ can be evaluated individually using the techniques of partial fractions, contour integration and conjugate complex functions.

\section{References}

[1] A.A. Griffith, Philos. Trans. R. Soc. 221, 163 (1921).

[2] I.N. Sneddon, M. Lowengrub, Crack Problems in the Classical Theory of Elasticity, Wiley, New York 1969.

[3] G.E. Tupholme, Mech. Res. Commun. 45, 48 (2012).

[4] G.E. Tupholme, Eng. Fract. Mech. 134, 451 (2015).

[5] D. Shechtman, I. Blech, D. Gratias, J.W. Cahn, Phys. Rev. Lett. 53, 1951 (1984).

[6] T.-Y. Fan, Z-Y. Tang, W-Q. Chen, Eng. Fract. Mech. 82, 185 (2012). 
[7] T.Y. Fan, The Mathematical Theory of Elasticity of Quasicrystals and Its Applications, Science Press, Springer-Verlag, Beijing 2011.

[8] T.Y. Fan, Engineering 5, 407 (2013).

[9] D.H. Ding, W.G. Yang, C.Z. Hu, R.H. Wang, Phys. Rev. B 48, 7003 (1993).

[10] T.P. Yadav, AIMS Mater. Sci. 4, 172 (2017).

[11] G. Altay, M.C. Dökmeci, Int. J. Solids Struct. 49 3255 (2012).

[12] X. Wang, E. Pan. Pramana J. Phys. 70, 911 (2008).

[13] C. Li, Y. Liu, Chin. Phys. 13, 924 (2004).

[14] L.-Z. Yang, Y. Gao, E. Pan, N. Waksmanski, Acta Phys. Pol. A 126, 467 (2014).

[15] X.Y. Li, P.D. Li, T.H. Wu, M.X. Shi, Z.W. Zhu, Phys. Lett. A 378, 826 (2014).

[16] J. Yu, J. Guo, E. Pan, Y. Xing, Appl. Math. Mech. 36, 793 (2015)

[17] J. Yu, J. Guo, Y. Xing, Chin. J. Aero. 28, 1287 (2015).

[18] L. Zhang, D. Wu, W. Xu, L. Yang, A. Ricoeur, Z. Wang, Y. Gao, Phys. Lett. A 380, 3222 (2016).

[19] J. Guo, E. Pan, ASME J. Appl. Mech. 83, 081007 (2016).

[20] C. Fan, Y. Li, G. Xu, M. Zhao, Mech. Res. Commun. 74, 39 (2016).
[21] J. Guo, Z. Zhang, Y. Xing, Phiplos. Mag. 96, 349 (2016).

[22] J. Yang, X. Li, Theor. Appl. Fract. Mech. 82, 17 (2016).

[23] G.E. Tupholme, Acta Mech. 228, 547 (2017).

[24] G.E. Tupholme, Meccanica 53, 973 (2018).

[25] J. Yang, Y. Zhou, H. Ma, S. Ding, X. Li, Int. J. Solids Struct. 108, 175 (2017).

[26] Y.-B. Zhou, X.-F. Li, Eng. Fract. Mech. 189, 133 (2018).

[27] J. Sladek, V. Sladek, E. Pan, Int. J. Solids Struct. 50, 3975 (2013).

[28] X.-Y. Li. Int. J. Solids Struct. 51, 1442 (2014).

[29] B.A. Bilby, J.D. Eshelby, Dislocations and the Theory of Fracture, in: Fracture, Ed. H. Liebowitz, Vol. 1, Academic Press, New York 1968, p. 99.

[30] R.W. Lardner, Mathematical Theory of Dislocations and Fracture, University of Toronto Press, Toronto 1974.

[31] G. Leibfried, Z. Phys. 130, 214 (1951).

[32] N.I. Muskhelishvili, Singular Integral Equations, Noordhoff Int. Pub., Leyden 1953.

[33] F.D. Gakhov, Boundary Value Problems, Pergamon, Oxford 1966. 